\title{
Echinoderms of the Dampier Archipelago, Western Australia
}

\author{
Loisette M. Marsh* and Susan M. Morrison \\ Department of Aquatic Zoology (Marine Invertebrates), Western Australian Museum \\ Francis Street, Perth, Western Australia 6000, Australia \\ email: *c/o jane.fromont@museum.wa.gov.au \\ sue.morrison@museum.wa.gov.au
}

\begin{abstract}
The results of two diving surveys (DA1/98 and DA3/99) and a dredge survey (DA2/99) conducted in the waters of the Dampier Archipelago, Western Australia, are summarised. The diving surveys sampled 70 sites in the eastern (DA1/98) and western (DA3/99) halves of the Archipelago. Considerable differences were demonstrated between the echinoderm faunas of these areas, with more species (139) recorded in western than eastern (115) areas and only 74 species in common between the two. There are major habitat differences between the eastern and western parts of the archipelago, with more areas of soft substrate in the western part, providing more habitat for astropectinid starfishes and burrowing heart urchins. The dredging survey (DA2/99), which sampled 100 sites spread throughout the archipelago, showed that the area has an extremely rich echinoderm fauna, unmatched by any other in Western Australia. Fifty-two percent of the species taken by dredging were not found on the dive surveys. A complete list of all species found during the diving and dredging expeditions (260) and a supplementary list of material from the Dampier Archipelago held in the Western Australian Museum are presented, making a total of 286 species, the highest number recorded from any part of Western Australia. This rich fauna is a reflection of the diversity of habitats and the range of exposure to wave action, turbidity and currents related to the complex topography of the archipelago. The archipelago is the type locality of three valid echinoderm species and two from deeper water north of the archipelago. The distribution of species falls roughly into five groups: $9 \%$ are endemic to northwestern Western Australia, with some extending into the Northern Territory; $11 \%$ are endemic to northern Australia including Queensland and some extending to New South Wales; $28 \%$ extend northward to Indonesia, with some to Japan; $47 \%$ are widespread Indo-West Pacific species; and $6 \%$ are Indian Ocean species extending to the Red Sea or Persian Gulf.
\end{abstract}

\section{INTRODUCTION}

The present collections are the result of three expeditions to the Dampier Archipelago, as part of the joint WA Museum-Woodside Energy Ltd. Partnership to survey the marine fauna of the Dampier Archipelago. The expeditions were designated as follows: DA1/98 (diving), DA2/99 (dredging) and DA3/99 (diving). Collections were also made by snorkelling and low tide collecting.

DA1/98 (October 1998) sampled 35 sites in the eastern half of the Archipelago - Delambre, Legendre, Dolphin, Angel and Gidley Islands, several smaller islands and Madeleine and Hamersley Shoals. The second diving expedition (DA3/99) sampled 35 sites in the western half of the Archipelago (East and West Lewis, Malus, Enderby, Rosemary and Kendrew Islands, as well as several smaller islands and rocks and some of the inter-island channels) in August-September
1999. The dredging survey (DA2/99) sampled 100 sites spread over the whole archipelago, between the islands, in Nickol Bay and some deeper sites seaward of the Archipelago in July 1999. Refer to the Station Lists section of this volume for the diving and dredging expedition data and maps.

Published records specifically from the Dampier Archipelago are few, dating mainly from the German Gazelle Expedition (Studer, 1880, 1882, 1884; A.H. Clark, 1909; Lampert, 1889) and Mjoberg's Swedish scientific expedition to Australia 1910-1913. However, most of the latter collections were from the Cape Jaubert area, further east (Mortensen, 1918; Ekman, 1918; Gislén, 1919). Material from the Dampier Archipelago is included in a number of taxonomic papers (A.H. Clark, 1912; Baker, 1980; McNamara, 1982, 1992, 1995; Rowe and Marsh, 1982; Rowe, 1985; Rowe and Pawson, 1985; Rowe et al., 1986; Hoggett, 1990, 1991), while 
the regional fauna is discussed in zoogeographic accounts (Marsh, 1976; Marsh and Marshall, 1983; Wilson and Allen, 1987).

There are extensive collections in the Western Australian Museum resulting from fieldwork by museum staff at Rosemary, Legendre and Delambre Islands (1961), Rosemary Island (1971), Rosemary and Kendrew Islands during1972-74 (Wilson and Marsh, 1974, 1975; Wilson et al., 1974), the Burrup Peninsula and nearby islands (Marsh, 1978) and from cruises by Davena (1960), Soela (1982) and Lady Basten (1995).

\section{MATERIALS AND METHODS}

\section{Diving expeditions (DA1/98, DA3/99)}

The locations of the stations of the 1998 and 1999 dive expeditions are shown on the station maps in the Station Lists section of this volume.

\section{DA1/98}

Echinoderms were collected from stations DA1/ 98/01-35 by walking at low tide, snorkelling in the shallows and by SCUBA diving to $24 \mathrm{~m}$. At many sites (DA1/98/01, 03, 04, 06, 08, 09, 12, 13, 15, 16, $18,19,21,22,24,26,27,29,30,32,33$ and 35) a transect line ( $25 \mathrm{~m}$ long) was laid on the contour and animals observed or collected within $5 \mathrm{~m}$ on each side of transect.

\section{DA3/99}

Echinoderms were collected from stations DA3/ $99 / 36-70$ by walking at low tide (DA3/99/38, 42, $45,48,51,54,59,62$ and 66 ) or diving (DA3/99/36, $37,39,40,41,43,44,46,47,49,50,52,53,55,56,57$, $58,60,61,63,64,65,67,68,69$ and 70$)$. A $25 \mathrm{~m}$ transect line was laid on the contour and animals observed or collected within $5 \mathrm{~m}$ on either side of the transect at each of the dive stations.

\section{Dredging expedition (DA2/99)}

Collections were made from 100 stations (DA2/ 99/01-100) by rake box dredge (88 stations), scoop box dredge (9), shovel box dredge (1) and grab (3). Depths ranged from 5-43 m; 15 stations were in the 5-10 m depth range, 42 in $11-20 \mathrm{~m}, 13$ in $21-30 \mathrm{~m}$ and 30 in $31-43 \mathrm{~m}$ (refer to the station maps section of this report).

\section{RESULTS}

Details of the echinoderm fauna are discussed more specifically in the results from the individual diving (DA1/98, DA3/99) and dredging (DA2/99) expeditions following this brief overview.

A total of 260 species of echinoderms was collected from the three surveys (Appendix 1) and a further 26 are recorded from collections in the Western Australian Museum (Appendix 2), bringing the total to 286. Doubtful species are excluded from this tally, but some of these may prove to be valid species and may be undescribed. This total is more than half again as many species as have been recorded from Ashmore Reef and Cartier Island (178 species), the next most speciose area off northwestern Australia (Marsh et al. 1993). However, the Dampier Archipelago figure is not surprising in view of the high habitat diversity of coral reefs in both clear and turbid water, rocky shores, sand and mud flats and mangroves. There is a range from clear to turbid water and from exposed to sheltered conditions, with a highly complex island coastline and strong tidal currents. The archipelago has also been more intensively sampled (170 sites) than anywhere else in Western Australia by dredging, diving, snorkelling and low tide collection.

The Ophiuroidea are the most speciose class, with 76 species recorded during the dredging and diving surveys and another 13 from previous records, giving a total of 89 . The next most speciose are the Holothuroidea with 66 species (surveys) and a further two (total 68). The Asteroidea are represented by 49 species plus five from previous records ( 54 total); the Crinoidea by 35 species with one additional ( 36 total); and the Echinoidea with 34 species plus five additional ( 39 total).

Mermaid Strait is the type locality for three currently recognised species of echinoderms and two which have fallen into synonymy, all collected by the German expedition on S.M.S. Gazelle, 18741876. The valid species are an ophiuroid, Dictenophiura stellata (Studer, 1882) and two holothurians, Loisettea gazellae (Lampert, 1889) and Plesiocolochirus dispar (Lampert, 1889). The latter was not found during the survey but the former two were found at a number of sites (Appendix 1). An ophiuroid, Ophiothrix smaragdina Studer, 1882 has the type locality northwestern Australia, $50 \mathrm{fms}$ (91 $\mathrm{m})$, probably north of the Dampier Archipelago. An echinoid, Nacospatangus interruptus (Studer, 1880), was also taken in $30 \mathrm{fms}(55 \mathrm{~m})$ by the Gazelle Expedition, probably north of the Dampier Archipelago. This species has rarely been collected before and is only known from coastal waters between the Dampier Archipelago and Lagrange Bay, south-west of Broome. Apart from the present collections it is known from few specimens, most from the Dampier area between 18 and $68 \mathrm{~m}$.

\section{Diving expeditions (DA1/98, DA3/99)}

\section{DA1/98}

One hundred and fifteen species were recorded. Table 1 lists the species, station numbers and depth 
range at which they were collected or observed within the transect and elsewhere. Table 2 lists the species collected or observed at each station.

\section{Crinoidea}

The crinoid fauna is rather depauperate. Only 13 species were collected and few species were common. This can be largely related to water turbidity as crinoids are more abundant on reefs further offshore in clear water. They were noticeably more plentiful in areas with strong currents and one species, Oligometra carpenteri, is only found on gorgonian corals, which also favour areas with good water movement.

\section{Asteroidea}

The asteroid fauna was also depauperate with only 14 species recorded. Two genera, Luidia and Astropecten, are characteristic of sandy or muddy substrates, while the four species of Ophidiasteridae were only found at the seaward sites. The small Linckia multifora was the most common of these, found at six sites and very common (29 recorded) at Madeleine Shoals.

The large cushion star, Culcita schmideliana, was nowhere abundant but was found at more sites (10) than any other asteroid. The coral predator, Acanthaster planci, was found at five sites, depth range $3.4-24.0 \mathrm{~m}$, but with only a single specimen at each site, except station DA1/98/04 where several were seen. All sites were on the outer part of the archipelago from Hamersley Shoal to Haüy Island.

Several uncommon species were found - Fromia sp. (which may be undescribed), Gomophia sphenisci and Echinaster superbus. A species of Asterina may also be undescribed.

\section{Ophiuroidea}

In contrast to the previous two classes, the ophiuroids were exceptionally diverse with 46 species, many of which were very common. However, the majority were small, cryptic species found among corals, in rock crevices and in sponges.

One species, Ophiodyscrita acosmeta, has only been found previously at two localities, both in the Kimberley. The present record is, thus, an extension of range. Critical taxonomic evaluation of some of the species of Ophiactis may show that some species should be synonymised, as suggested by Clark and Rowe (1971) or, with more material now available, that more species should be recognised. Several species remain unidentified.

\section{Echinoidea}

The echinoid fauna is rather small with only 15 species found, some of which were only represented by dead tests or fragments. There are nine species of regular urchins; two of sand dollars and four of heart urchins. The most abundant was the needle-spined urchin, Diadema setosum, found at 17 stations with 129 found on the transect at station DA1/98/04 and 60 at DA1/98/15. The small, rockboring urchin, Echinostrephus molaris, was found at 10 sites with up to 15 at one site. The small sand dollar, Peronella orbicularis, was found at eight sites and the heart urchin, Breynia desorii, at six but both species were represented almost entirely by dead tests. All the echinoid species are common in northwestern Australia and some are widespread Indo-West Pacific species (Clark and Rowe, 1971).

\section{Holothuroidea}

Twenty seven species of holothurians were found. The most common were Holothuria (Halodeima) atra from 17 sites and $H$. (H.) edulis and $H$. (Mertensiothuria) leucospilota, each from 12 sites. Six was the highest number of species recorded from any transect although more were observed but not counted at some non-transect stations. A few specimens could not be identified as they were inadvertently preserved in formalin, which dissolved the diagnostic spicules in the body wall. Most of the holothurians are widespread Indo-West Pacific species but several, characteristic of offshore coral reefs, were not present in the areas sampled.

\section{DA3/99}

One-hundred and thirty nine species were recorded from the western part of the Dampier Archipelago by the DA3/99 diving expedition, compared with 115 from the survey of the eastern islands (DA1/98). Of the species collected, 74 (54\%) were in common between the two areas. Table 3 lists the species, station numbers and depth range at which they were collected. Table 4 lists the species collected or observed at each station.

\section{Crinoidea}

Twenty two crinoid species were recorded, 13 of which were not found in the DA1/98 diving survey and only nine were in common between the eastern and western parts of the archipelago. All but three were found subtidally. Only two species could be regarded as locally common with 400 examples of Oligometra carpenteri on gorgonians and 32 Amphimetra tessellata on the transect at $17 \mathrm{~m}$ in the channel between Enderby and West Lewis Islands (station DA3/99/55). Seven species of crinoids were recorded at this station while eight were found south west of Enderby Island at $14 \mathrm{~m}$ (DA3/99/65). However, most species at most sites are recorded as one or two individuals. Only two species, Comanthina variabilis and Clarkcomanthus littoralis, were found at four of the 35 sites, while four species were found at three sites. The remainder were only collected from one or two stations. Apart from species that live in the crevices of intertidal coral 
reefs, crinoids favour deeper subtidal areas with good water flow and moderately clear water.

\section{Asteroidea}

Thirty species of asteroids were recorded from the western half of the archipelago, more than double the number (14) recorded from the eastern half by the DAI/98 diving expedition. Thirteen species were in common between the two areas. Three species of Astropecten, not found in 1998, were collected from sand flats at Malus and Enderby Islands. Only one species, Linckia multifora, found at eight subtidal stations with up to 10 in one transect at Bare Rock (station DA3/99/50), could be regarded as common. Protoreaster nodulosus, although only found at two sites, was common on the sand flats at Enderby Island (station DA3/99/51). Other moderately common species are Culcita schmideliana found at 13 sites (from intertidal to $17 \mathrm{~m}$ ) and Pseudoreaster obtusangulus at five intertidal and shallow sites, but both were in low numbers at each site.

The coral predator, Acanthaster planci, was found at nine sites, between 5-17 m, but was in low numbers with three the highest number seen at one station. No "outbreaks" were found and all those seen were in the outer parts of the archipelago, from Bare Rock to Nelson Rocks. The distribution is similar to that found in the early 1970's (Wilson and Marsh 1974) when large populations were found at Kendrew and Rosemary Islands. Other species of asteroids were recorded at one or two stations with one or two specimens at each.

\section{Ophiuroidea}

Thirty eight ophiuroid species were recorded from the DA3/99 dive survey, six fewer than the 44 recorded from the eastern part of the Archipelago by the DA1/98 dive survey. Twenty six species were in common between the two surveys. Species found at the most stations were: Ophiactis savignyi, a small species found in rock or sponges (11 sites); Macrophiothrix 'caenosa' (nine sites); Ophiothrix martensi (six sites); Ophiothrix smaragdina (five sites); Ophiothrix ciliaris common at 16 sites, usually in sponges; O. exigua and $O$. plana, both at nine sites; Ophioplocus imbricatus (seven sites); Ophiarachnella gorgonia and Ophionereis intermedia (six sites); and O. dubia at five. In 1998, five species in the family Ophiocomidae were found but only one in 1999. Most ophiocomids are fairly large brittle stars, found on rock platforms or coral reefs, so their absence is puzzling. Many of the ophiuroids are small, cryptic species found in sponges and rock crevices, while the larger ones are usually concealed under rocks during the day and so are not a conspicuous part of the fauna.

\section{Echinoidea}

Twenty echinoid species were recorded from the western half of the archipelago by the DA3/99 diving expedition and ten of these are in common with the DA1/98 diving expedition. By far the most abundant species is the needle-spined urchin, Diadema setosum, found at 15 stations (intertidal to $17 \mathrm{~m}$ ), at nine of which it was common with the greatest number (101) in the transect at station DA3/99/53 (Enderby Island). Another common species was the rock-burrowing urchin, Echinostrephus molaris, found at seven subtidal stations, from 5-17 $\mathrm{m}$ and common at three sites. Of great interest was the record from Kendrew Island (DA3/99/46) of Phyllacanthus imperialis, a coral reef species not previously recorded in Western Australia although it is found on the Great Barrier Reef, Queensland. Curiously, no members of the family Toxopneustidae, represented by three species in the 1998 survey, was found. Heart urchins (Loveniidae and Schizasteridae) characteristic of soft substrates were represented by three species in 1998 and by two species in 1999. However, three additional families, Fibulariidae, Brissidae and Spatangidae, were represented by six species one of which, Nacospatangus interruptus, is endemic to the north-west coast from the Dampier Archipelago to Lagrange Bay.

\section{Holothuroidea}

Twenty nine species of holothurians were recorded from the DA3/99 survey, one more than the DA1/98 survey; 16 species are in common between the two surveys. Holothurians were more abundant than other classes of echinoderms in the survey: Holothuria leucospilota was found at 14 stations, common at two intertidal sites; Holothuria atra at 11 but common at only one; Stichopus chloronotus at six, common at three with 16 individuals in a transect at station DA3/99/44 (Rosemary Island); Actinopyga echinites at nine sites, common at three with 28 recorded at DA3/99/47 (Kendrew Island) and Synaptula recta at six and common at one. Other moderately common species were: Holothuria hilla (five sites, common at one); Holothuria impatiens (six, common at one); Holothuria cinerascens, although only found at two, was common at one; and Holothuria scabra, only found at one site where there were many (uncounted).

\section{Dredging expedition (DA2/99)}

Echinoderms were found at all but 12 of the 100 stations and all five classes were taken at 18 stations (DA2/99/04, 06, 07, 09, 10, 12, 18, 21, 23, 33, 37, 41, $44,49,57,62,68$ and 69). The locations of the stations are shown on the station maps in the Station Lists section of this Volume.

The dredge survey revealed a far richer fauna than was sampled by the dive surveys, partly because it sampled deeper water but also because many infaunal species not readily collected by diving were found. In all, 170 species were collected 
or observed. Of these, 54 (31\%) were in common with the first diving expedition (DA1/98), 65 (38\%) in common with the second diving expedition (DA3/99), 42 (25\%) of the dredged species were in common with both dive trips while $89(52 \%)$ of the species were only taken by dredging (DA2/99). Table 5 lists the species, station numbers and depth range at which they were collected.

\section{Crinoidea}

A total of 19 crinoid species was collected. Of these, six were in common with DA1/98 and 10 with DA3/99, six were in common with both dive trips and nine were not taken on either dive survey. The collection included several species associated with gorgonians and one, Comatula rotalaria, found on soft substrates.

\section{Asteroidea}

Thirty one species of asteroids were collected. Of these, only five were in common with DA1/98 and 12 with DA3/99. Five were in common with both dive surveys and 19 were not taken on either dive survey. At least 14 species are always found on soft substrates and several others are found on mixed hard and soft bottom.

The most common species, Stellaster equestris, was found at 17 stations, with over 100 recorded from DA2/99/18. The species recorded at most stations (21) was Astropecten zebra with $A$. velitaris a close second (20). Luidia hardwicki and Metrodira subulata were found at 14 stations, being abundant at DA2/ $99 / 18$. Most of the species have also been collected from the North-West Shelf, but a few are only known from fairly shallow coastal waters.

\section{Ophiuroidea}

There is a rich ophiuroid fauna, with 50 species recorded from the DA2/99 dredge survey. Of these, 22 are in common with DA1/98, 20 with DA3/99 and 17 with both dive surveys, while 22 were only collected on the dredge survey (DA2/99). The most common was Ophiothrix ciliaris, usually associated with sponges; this was taken at 34 stations. Other common species in order of the number of stations at which they were collected are: Ophiochasma stellata (19), Macrophiothrix megapoma (19), Ophiactis savignyi (17), Ophiothrix smaragdina (17), Macrophiothrix melanosticta and Ophiothrix martensi (14). Two species of Ophiarachnella appear to be undescribed; one of these was taken at 12 stations. Several species of uncertain identity may be new records for Australia.

Two species of brittle stars have their type locality in or near the Dampier Archipelago. These are Ophiothrix smaragdina Studer, 1882, first collected by the Gazelle expedition north of the Dampier Archipelago and Dictenophiura stellata (Studer, 1882) also first collected by the Gazelle Expedition, from Mermaid Strait. Ophiothrix smaragdina is endemic to northwestern Australian coasts, from the Dampier Archipelago to Darwin, while the range of Dictenophiura stellata extends northward to Indonesia.

\section{Echinoidea}

The echinoid fauna of 25 species includes 15 of sand dollars and heart urchins, reflecting the soft substrates sampled. Eleven species are in common with diving expedition DA1/98 and 10 with DA3/ 99. Five species were also taken on both dive surveys and 10 were not found on either dive survey. Several are of particular interest. The type locality of Nacospatangus interruptus, first collected by the Gazelle Expedition (1874-76), had its type locality recorded as Western Australia (Studer 1880) but, as the Gazelle only sampled near Shark Bay and in and north of the Dampier Archipelago, it is probable that the Dampier Archipelago is the correct locality as it has never been collected in Shark Bay. This is an endemic species found only between the Dampier Archipelago and LaGrange Bay and is considered rare. However, it is not uncommon in the Dampier Archipelago, where it was found at eight stations from depths of 7-33 m during the dredge survey and it was also taken during the diving expedition DA3/99. A single specimen of a species of Brissopsis, nearest to $B$. persica from the Persian Gulf, may be undescribed.

\section{Holothuroidea}

Dredging uncovered a rich holothurian fauna, including many infaunal species, making a total of 45. Of these, 11 are in common with the first diving survey (DA1/98), 14 with the second (DA3/99) and nine with both. Twenty-nine species taken by dredging were not found on either of the diving surveys.

Species found at most stations were Cercodemas anceps, Colochirus quadrangularis and Synaptula recta, each at 12 stations and the infaunal Acaudina leucoprocta at nine. A single specimen of Loisettea gazellae was collected. This was first collected by the Gazelle Expedition, from Mermaid Strait which is the only known locality of this species in Australia (Lampert, 1889). The only other record is from Sumbawa in the Lesser Sunda Islands, Indonesia. Loisettea amphictena, found at seven stations, is endemic to the inner continental shelf of northwestern Australia, from Shark Bay to Darwin.

Holothuria (Metriatyla) timana is a new record for coastal waters. It has been found at Ashmore Reef, Timor Sea and is known from the Great Barrier Reef. Two endemic northern Australian species, Leptopentacta grisea and Phyllophorus spiculata, have their ranges extended from Broome to the Dampier Archipelago and Plesiocolochirus challengeri and Molpadia scabrum have ranges extended from the Northern Territory to the Dampier Archipelago. The range of Stolus canescens is extended from the 
Great Barrier Reef westward to Dampier and it is also found in Indonesia. Two species in the Phyllophoridae could not be placed in a genus or species and may be undescribed.

\section{Biology}

Several species of ophiotrichid brittle stars were found living commensally with crinoids, perhaps semi-parasitically: Ophiomaza cacaotica was found on the comasterid feather stars Comanthina variabilis, C. nobilis and Comatula solaris; Macrophiothrix melanosticta was found associated with Comatula rotalaria and Gymnolophus obscura with Comanthus parvicirrus. Two species of ophiactids were found on sand dollars - Ophiodaphne formata on Peronella lesueuri and O. scripta on Echinodiscus auritus.

The soft substrata in the archipelago are rich in sand dollars (eight species of three families) and heart urchins (11 in six) (Appendix 1). Several sites in the archipelago had particularly high numbers of echinoderms (Tables 6, 7). The numbers of echinoderm species found at each diving (DA1/98, DA3/99) and dredging (DA2/ 99) site are listed (Tables 8,9 ). All these sites have a mixed habitat, usually with sand, algae and some corals. At the deeper sites with gorgonians several species of crinoids cling to the gorgonians, while several species of ophiotrichid brittle stars live in the cavities of sponges. Other species of echinoderms shelter under rock or dead coral slabs, while few are associated with living hard corals. The species tables of the reports of the individual expeditions indicate species found in coral, rock crevices and sponges (Tables 1, 3, 6, 7).

\section{Zoogeography}

The distribution of 265 echinoderms species found in the Dampier Archipelago falls roughly into five groups: those species endemic to northwestern Western Australia, some extending into the Northern Territory (23 species, $8.68 \%$ ); those endemic to northern Australia extending into Queensland, sometimes to NSW (29 species, $10.94 \%)$; those with their range extending northward to Indonesia with some to Japan (74 species, $27.92 \%$ ); more or less widespread IndoWest Pacific species (124 species, 46.79\%) and those found in the Indian Ocean, some extending to the Red Sea or Persian Gulf (15 species, 5.66\%). Widespread Indo-West Pacific species make up the largest group followed by those with a southeast Asian distribution. However, a significant group $(8.7 \%)$ are endemic to northwestern Australia, some only from the Dampier Archipelago to the Kimberley or even shorter distances e.g. Nacospatangus interruptus, known only from the Dampier Archipelago to LaGrange Bay.
Phyllacanthus imperialis has not previously been recorded from northwestern Australia. It is an IndoPacific species also recorded from the Great Barrier Reef, Queensland. The range of Ophiodyscrita acosmeta has been extended from the Kimberley and Broome to Dampier. Two species of Asteroidea (Fromia sp. and Asterina sp.) are believed to be undescribed, while several species of Ophiuroidea may be undescribed (Ophiarachnella sp. and Ophiothrix sp.). The Ophiactidae and Amphiuridae are in need of revision before some species can be determined. Some ophiuroids, tentatively identified, have not been recorded previously from Australia.

\section{DISCUSSION}

The diving expeditions documented considerable differences in the echinoderm fauna of the eastern and western parts of the archipelago, with more species recorded in the western areas - 139 in the western part and 115 in the eastern part. Of these, only 74 species $(54 \%)$ are in common between the two areas. Some differences may be due to the choice of sampling sites, which may reflect localised habitat differences. However, there are major habitat differences between the eastern and western parts of the archipelago, with more areas of soft substrate in the western part, providing more habitat for astropectinid starfishes and burrowing heart urchins.

The wide coverage of the dredge survey and the intensity of sampling have shown that the area has an extremely rich echinoderm fauna, unmatched by any other area in Western Australia. However, it is now the most intensively sampled area, so accurate comparisons cannot be made with other areas. The fact that $52 \%$ of the species taken by dredging were not found on the dive surveys suggests that there may be yet more species to be found in the Archipelago.

The Dampier Archipelago has proved to be an exceptionally rich area for echinoderms, largely because of its diverse range of hydrology and habitats around a variety of island types. Particularly rich areas (dive sites) are in the channels between islands, where there is a strong current e.g. DA1/98/22 (off Delambre Island), DA3/99/45 (Tish Reef, Rosemary Island) and DA3/99/65 (off the SW corner of Enderby Island). The richest dredge sites were DA2/99/06 ( $\mathrm{N}$ of Legendre Island), DA2/99/75 (E of Goodwyn Island), DA2/99/04 (NW of Legendre Island) and DA2/99/09 ( $\mathrm{N}$ of Delambre Island).

It is vital that representative areas of each habitat type be preserved from industrial development and it is unfortunate that the Dampier Archipelago was chosen for development before its faunal richness was discovered. 
Notes on species recorded from Australia for the first time and on other species of interest.

\section{Asteroidea}

Fromia sp. ? nov. This species was earlier thought to be $F$. zodiacalis by Marsh (illustrated in Coleman 1994) despite the fact that one of the specimens in the type series was synonymised by Perrier (1875) with Scytaster aegyptiacus (now Gomophia egyptiaca Gray, 1840). It is now believed to be an undescribed species endemic to northwestern Australia, from Exmouth Gulf to Port Hedland. Asterina sp., represented by a single specimen, may be undescribed.

\section{Ophiuroidea}

Amphiura (Amphiura) morosa Koehler, 1905. Previously known from Indonesia it is, therefore, not surprising to find it in northwestern Australia.

Amphiura (Amphiura) phrixocantha Clark and Clark, 1976. Rowe and Gates (1995) include this in the synonymy of $A$. (A.) maxima Lyman, 1879 following Baker (1979). I have examined Baker's specimen and found it is not conspecific with specimens from Dampier nor with $A$. phrixocantha based on description of the holotype. Therefore, I prefer to retain the name A. phrixocantha for the Western Australian specimens which closely match the original description.

Ophiactis sp. cf. brachyura Döderlein, 1898. The specimens are closer to this species than to any Ophiactis previously recorded from Australia.

Ophiactis versicolor H. L. Clark, 1939. This Red Sea species was included in the synonymy of $O$. savignyi (Müller and Troschel, 1842) by Cherbonnier and Guille (1978). However the present specimens differ significantly from $O$. savignyi and fit the description of $O$. versicolor.

Ophiodaphne scripta (Koehler, 1904). This species was considered to be a possible synonym of Ophiodaphne materna Koehler, 1930 by Cherbonnier and Guille, 1978. However, Guille (1981) showed that $O$. materna is a junior synonym of $O$. formata (Koehler, 1905) and that O. scripta (Koehler, 1904) is a valid species, here recorded from Australia for the first time.

Ophiothrix (Ophiothrix) sp. cf. comata Müller and Troschel, 1842. The Dampier specimens are closer to this species than to any species so far recorded from Australia.

Ophiarachnella megaloplax Bell, 1884. This species was erroneously included in the synonymy of
O. gorgonia (Müller and Troschel, 1842) by Rowe and Gates 1995 (Rowe pers. comm. 2001) and so is here re-instated as a valid species.

Ophiarachnella sp. 1. May represent an undescribed species.

\section{Echinoidea}

Phyllacanthus imperialis (Lamarck, 1816) has been recorded from the Great Barrier Reef but not as yet from the reefs off northwestern Australia. It was surprising, therefore, to find a specimen in the Dampier Archipelago and it should probably be regarded as a vagrant.or chance occurrence.

Temnopleurus decipiens (de Meijere, 1904) has not been previously recorded from Australia but is known from Indonesia, so the occurrence of this species at Dampier is not surprising.

Schizaster compactus (Koehler, 1914) was first recorded from Australia by McNamara (1995) from specimens taken north of Dampier and from near Rosemary Island in the Dampier Archipelago. The latter specimen was recorded by McNamara and Philip (1980) and Rowe and Gates (1995) as Schizaster (Schizaster) lacunosus (Linnaeus) and re-identified when further material became available.

Brissopsis sp. aff. persica Mortensen, 1940. This may be an undescribed species, very close to $B$. persica from the Persian Gulf.

\section{Holothuroidea}

Stichopus cf. noctivagus Cherbonnier, 1980. This species has not previously been recorded from Australia but the specimen fits the description of this species.

\section{REFERENCES}

Baker, A.N. (1980). Euryalinid Ophiuroidea (Echinodermata) from Australia, New Zealand and the south-west Pacific Ocean. New Zealand Journal of Zoology 7: 11-83.

Cherbonnier, G. and Guille, A. (1978). Faune de Madagascar 48, Echinodermes: Ophiurides. Editions du C. N. R. S. Paris. 272 pp.

Clark, A.H. (1909). The crinoids of the Gazelle Expedition. Zoologischer Anzeiger 34: 363-370.

Clark, A.H. (1912). The crinoids of the Museum fuer Naturkunde, Berlin. Proceedings of the U.S. National Museum 43: 381-410.

Clark, A.M. and Rowe, F.W.E. (1971). Monograph of Shallow-water Indo-West Pacific echinoderms. London: Trustees of the British Museum (Natural History). 238 pp.

Ekman, S. (1918). Results of Dr E. Mjoberg's Swedish Scientific expedition to Australia, 1910-1913. 19. 
Holothurioidea. Kungliga Svenska Vetenskapsakademiens Fordhandlingar 58: 1-70.

Gislén, T. (1919). Results of Dr E. Mjoberg's Swedish Scientific expedition to Australia, 1910-1913. 23. Crinoids. Kungliga Svenska Vetenskapsakademiens Fordhandlingar 59: 3-37.

Guille, A. (1981). Echinodermes: Ophiurides. Résultats des Campagnes Musorstom 1. Philippines (18-28 Mars 1976): 413-456.

Hoggett, A.K. (1990). Taxonomy and systematic position of the brittle-star genus Macrophiothrix H.L. Clark (Echinodermata: Ophiuroidea). Unpublished $\mathrm{Ph} \mathrm{D}$ thesis, University of Queensland, St Lucia.

Hoggett, A.K. (1991). The genus Macrophiothrix (Ophiuroidea: Ophiotrichidae) in Australian waters. Invertebrate Taxonomy 4: 1077-1146.

Lampert, K. (1889). Die Während der Expedition SMS Gazelle 1874-1876 von Prof. Dr Th. Studer gesammelten Holothurien. Zoologische Jahrbiicher (Systematik) 4: 806-858.

McNamara, K.J. (1982). Taxonomy and evolution of living species of Breynia (Echinoidea: Spatangoida) from Australia. Records of the Western Australian Museum 10: 167-197.

McNamara, K.J. (1992). Geographical and stratigraphical distribution of the echinoid Echinometra mathaei (Blainville) in Western Australia. Records of the Western Australian Museum 16: 79-86.

McNamara, K.J. (1995). The spatangoid echinoid Schizaster (Schizaster) compactus (Koehler, 1914) in Western Australia. Records of the Western Australian Museum 17: 315-323.

McNamara, K. J. and Philip, G. M. (1980). Living Australian schizasterid echinoids. Proceedings of the Linnean Society of New South Wales 104: 127-146.

Marsh, L.M. (1976). Western Australian Asteroidea since H. L. Clark. Thalassia Jugoslavica 12: 213-225.

Marsh, L.M. (1978). Part IV. Report on the corals and some associated invertebrates of the Dampier Archipelago: 1-67. In Hutchins, J.B., Slack-Smith, S.M. and Marsh, L.M. (eds), Report on the Marine Fauna and Flora of the Dampier Archipelago. Western Australian Museum, Perth. Unpublished reports submitted to Meagher and LeProvost, Consultant Biologists, December, 1978.

Marsh, L.M. and Marshall, J.I. (1983). Some aspects of the zoogeography of northwestern Australian echinoderms (other than holothurians). Bulletin of Marine Science 33: 671-687.

Marsh, L.M., Vail, L.L., Hoggett, A.K. and Rowe, F.W.E. (1993). Part 6. Echinoderms of Ashmore Reef and Cartier Island. In Berry, P.F. (ed.), Marine Faunal Surveys of Ashmore Reef and Cartier Island, northwestern Australia. Records of the Western Australian Museum Supplement 44: 53-65.
Mortensen, T. (1918). Results of Dr E. Mjöberg's Swedish scientific expedition to Australia, 1910-1913. 21. Echinoidea. Kungliga Svenska Vetenskapsakademiens Fordhandlingar 58: 1-22.

Rowe, F.W.E. (1985). Six new species of Asterodiscides A.M. Clark (Echinodermata: Asteroidea), with a discussion of the origin and distribution of the Asterodiscididae and other amphi-Pacific echinoderms. Bulletin du Muséum national d'Histoire naturelle, Paris, Séries 4, 7, Section A (3): 531-577.

Rowe, F.W.E., Hoggett, A.K., Birtles, R.A. and Vail, L.L. (1986). Revision of some comasterid genera from Australia (Echinodermata: Crinoidea), with the description of two new genera and nine new species. Journal of the Linnean Society (Zoology) 86: 197-277.

Rowe, F.W.E. and Marsh, L.M. (1982). 6. A revision of the asterinid genus Nepanthia Gray, 1840 (Echinodermata: Asteroidea), with the description of three new species. Australian Museum Memoir 16: 89-120.

Rowe, F.W.E. and Pawson, D. (1985). Loisettea amphictena, new genus, new species, from the sublittoral of northwestern Australia (Echinodermata: Holothuroidea). Proceedings of the Biological Society of Washington 98: 672-677.

Studer, T. (1880). Übersicht über die während der reise SMS Corvette Gazelle um die erde 1874-76 gesammelten Echinoiden. Monatsbericht der Koniglich Preussischen Academie der Wissenschaften zu Berlin 1880: 861-885.

Studer, T. (1882). Übersicht über die Ophiuriden, welche während der reise SMS 'Gazelle' um die erde 1874-76 gesammelten wurden. Abhandlungen Preussische Akademie der Wissenschaften 1882: 1-37.

Studer, T. (1884). Verzeichniss der während der reise SMS 'Gazelle' um die erde 1874-76 gesammelten Asteriden und Euryaliden. Abhandlungen Preussische Akademie der Wissenschaften 1884: 1-64.

Wilson, B.R. and Allen, G.R. (1987). Major components and distributions of marine fauna. pp 43-68. In Dyne, G.R. and Walton, D.W. (eds), Fauna of Australia. 2. General articles. Canberra Australian Government Publishing Service, Canberra. Volume 1A.

Wilson, B.R. and Marsh, L.M. (1974). Acanthaster studies on a Western Australian coral reef. In Proceedings of the Second International Symposium on Coral Reefs. Great Barrier Reef Committee, Brisbane, Queensland, 1973 1: $621-630$.

Wilson, B.R. and Marsh, L.M. (1975). Seasonal behaviour of a 'normal' population of Acanthaster in Western Australia. pp 167-179. In Crown-of-Thorns Starfish Seminar Proceedings, Brisbane, 6 September 1974. Australian Government Publishing Service, Canberra.

Wilson, B.R., Marsh, L.M. and Hutchins, J.B. (1974). A puffer fish predator of Crown-of-Thorns in Australia. Search 6: 601-602. 
Table 1 Echinoderms from the Dampier Archipelago collected by the first diving expedition (DA1/98); species, station numbers and depth range at which they were collected or observed within the transect and elsewhere. ( $\mathrm{c}=$ from coral; $\mathrm{r}=$ from rock crevice; $\mathrm{t}=$ collected on transect; $\mathrm{d}=$ dead test; $\mathrm{s}=$ from sponge; $\mathrm{v}=$ visual or photographic record).

\begin{tabular}{llll}
\hline & Intertidal & Depth 0-10 $\mathrm{m}$ & Depth $>\mathbf{1 0} \mathrm{m}$ \\
\hline ECHINODERMATA &
\end{tabular}

\section{Class CRINOIDEA}

Family Comasteridae

cf. Clarkcomanthus littoralis (Carpenter, 1888)

Comanthina variabilis (Bell, 1882)

Comanthus wahlbergi (Müller, 1843)

Comatula purpurea (Müller, 1843)

Oxycomanthus sp.

10,25

$13 t$

33

$29 \mathrm{t}$

$29 \mathrm{t}$

10

Family Zygometridae

Zygometra punctata A.H. Clark, 1912

Family Mariametridae

Lamprometra palmata (Müller, 1841)

Stephanometra indica (Smith, 1876)

Stephanometra oxyacantha (Hartlaub, 1890)

Family Colobometridae

Colobometra perspinosa (Carpenter, 1881)

Oligometra carpenteri (Bell, 1884)

Petasometra helianthoides A.H. Clark, 1912

Family Antedonidae

Dorometra parvicirra (Carpenter, 1888)

\section{Class ASTEROIDEA}

Family Luidiidae

Luidia maculata Müller \& Troschel, 1842

Family Astropectinidae

Astropecten sumbawanus Döderlein, 1917

Family Goniasteridae

Iconaster longimanus (Möbius, 1859)

Family Oreasteridae

Culcita schmideliana (Retzius, 1805)

Gymnanthenea globigera (Döderlein, 1915)

Pseudoreaster obtusangulus (Lamarck, 1816)

Family Ophidiasteridae

Fromia indica (Perrier, 1869)

Fromia sp. ?nov.

Gomophia sphenisci (A.M. Clark, 1967)

Linckia multifora (Lamarck, 1816)

Family Asterinidae

Asterina sp. ?nov.

11

2,23

33

33

9,16

25

16,22

12

30

26

$27 \mathrm{t}$

$15 t, 26,27 t$
$10 \mathrm{v}, 14 \mathrm{v}, 17 \mathrm{v}, 25 \mathrm{v}$

$$
7
$$

11,14
$3 \mathrm{v}, 16 \mathrm{v}, 33 \mathrm{t}, \mathrm{v}$ 16

$\begin{array}{ll} & 21,26 t, 27 t, v, 32 t, v \\ & 15 t \\ & 15 t, 26 t \\ 3 t \quad 4,15 t, 21,26 t, 27\end{array}$

$4 v, 27 \mathrm{v}, 32 \mathrm{t}, \mathrm{v}$
Family Acanthasteridae

Acanthaster planci (Linnaeus, 1758)

Family Echinasteridae

Echinaster superbus H.L. Clark, 1916

Echinaster varicolor H.L. Clark, 1938

\section{CLASS OPHIUROIDEA}

\section{Family Amphiuridae}

Amphioplus didymus H.L. Clark, 1938

Amphioplus (Amphichilus) ochroleuca (Brock, 1888)

Amphipholis squamata (Delle-Chiaje, 1828)

Amphiura bidentata H.L. Clark, 1938
4

$$
16 \mathrm{v}, 18 \mathrm{t}, \mathrm{v}
$$

$4 v, 26 t, v, 32$
33

$3 \mathrm{t}$

$29 t$

1
$32 \mathrm{~s}, \mathrm{t}$ 
$3 \mathrm{t}$

6,33

$1,3,13,33$

8

$29 t, 33 t$

$33 \mathrm{r}$

Amphiura diacritica H.L. Clark, 1938

Amphiura duncani Lyman, 1882

Amphiura velox Koehler, 1910

Amphiura sp.

Dougaloplus echinatus (Ljungman, 1867)

Ophiocentrus dilatata (Koehler, 1905)

Ophiocentrus sp. aff. dilatata (Koehler, 1905)

Family Ophiactidae

Ophiactis fuscolineata H.L. Clark, 1938

Ophiactis luteomaculata H.L. Clark, 1915

Ophiactis macrolepidota Marktanner-

Turnertscher 1887

Ophiactis modesta Brock, 1888

Ophiactis sp. cf. modesta Brock, 1988

Ophiactis savignyi (Müller \& Troschel, 1842)

Ophiactis cf. savignyi (Muller \& Troschel, 1842)

Ophiactis sp.

Family Ophiotrichidae

Macrophiothrix 'caenosa' sensu Hoggett, 1990

Macrophiothrix callizona H.L. Clark, 1938

Macrophiothrix lineocaerulea H.L. Clark, 1928

Macrophiothrix lorioli A.M. Clark, 1968

Macrophiothrix megapoma H.L. Clark, 1938

Macrophiothrix paucispina Hoggett, 1991

Macrophiothrix sp. juv.

Ophiomaza cacaotica Lyman, 1871

Ophiothela danae Verrill, 1869

Ophiothrix (Keystonea) martensi Lyman, 1874

Ophiothrix (Keystonea) smaragdina Studer , 1882

Ophiothrix ciliaris (Lamarck, 1816)

Ophiothrix cf. ciliaris (Lamarck, 1816)

Ophiothrix exigua Lyman, 1874

Ophiothrix plana Lyman, 1874

Ophiothrix sp.

Family Ophiocomidae

Ophiocoma dentata Müller \& Troschel, 1842

Ophiocoma sp. cf. pusilla (Brock, 1888)

Ophiocomella sexradia (Duncan, 1887)

Ophiomastix mixta Lütken, 1869

Ophiomastix variabilis Koehler, 1905

Family Ophionereididae

Ophionereis dubia (Müller \& Troschel, 1842)

Ophionereis intermedia A.M. Clark, 1953

Ophionereis semoni (Döderlein, 1896)

Family Ophiodermatidae

Ophiarachnella gorgonia (Müller \& Troschel, 1842)

Ophiarachnella infernalis (Müller \& Troschel, 1842) 20

Ophiarachnella sphensci (Bell, 1894)

20

Ophiodyscrita acosmeta H.L. Clark, 1938

Family Ophiuridae

Ophiolepis unicolor H.L. Clark, 1938

Ophioplocus imbricatus (Müller \& Troschel, 1842)

14

11

10

$31 \mathrm{~s}$

10

10

14

10
$2,7 s, 17,23$

$10,28,31$

$2 s, 5,10,17,20,23$,

$25 \mathrm{~s}, 31,34$

$31 \mathrm{r}$

$10,23,31$
25,31
31
$31 \mathrm{r}$
23
$10,11,14,23$

$10 \mathrm{~s}, 23,31$

$5,7 \mathrm{~s}, 10 \mathrm{~s}, 17 \mathrm{c}, \mathrm{s}$,

$25,31 \mathrm{c}$

$5 \mathrm{~s}, 7 \mathrm{~s}, 10 \mathrm{~s}, 25 \mathrm{~s}, 31 \mathrm{~s}$

$5 \mathrm{~s}, 7 \mathrm{~s}, 10,11 \mathrm{~s}, 17$

$20 \mathrm{~s}, 23,25,31 \mathrm{r}, \mathrm{s}$

10,14

\section{Class ECHINOIDEA}

Family Cidaridae

Phyllacanthus longispinus Mortensen, 1918
$6,8,9,12,13,16 t, 29 t$

$22 \mathrm{~s}$

$33 t$

13

$1,3 \mathrm{t}, 6,8 \mathrm{~s}, 12,13$,

$16 t, 22,29 t, 33 t, 35$

$22 \mathrm{~s}$

1s, $22 \mathrm{~s}$

$3 t, 12,22,35$

$8 \mathrm{t}$

$3 c$

6

9,19

$3 t, 6 r, 8$

8,13

12

$1,3 t, s, 6 s, 8,9,12 t$,

$13 \mathrm{~s}, 19 \mathrm{~s}, 22 \mathrm{~s}, 29 \mathrm{t}, 33 \mathrm{t}, \mathrm{s}$

$22 \mathrm{~s}$

$3 \mathrm{t}, 6 \mathrm{~s}, 12,19 \mathrm{~s}$

$6,8 \mathrm{r}, \mathrm{s}, 12 \mathrm{t}, 13,16 \mathrm{t}$,

$19 \mathrm{~s}, 22 \mathrm{~s}, 29 \mathrm{t}, 33 \mathrm{t}$

1

16

16

1

1,22

$1,29 \mathrm{t}$

22

$6 \mathrm{~s}$

8,22

8

16

$8,12,33$

22

$6 s, 8,16$

4

21
$27,32 \mathrm{~s}, \mathrm{t}$

$4,15 t, 26,27 t, 32 s, t$
$32 t$

30

$15 t, s, 27 t$

$15 \mathrm{t}, \mathrm{s}, 27 \mathrm{t}$

$15 \mathrm{~s}$

$4,15 t, s, 21 s, 26,27 t$

$32 t, s$

$27 \mathrm{~s}$

$15 \mathrm{t}, \mathrm{s}, 26,27 \mathrm{t}$ 
Intertidal

Family Diadematidae

Diadema setosum (Leske, 1778)

Family Temnopleuridae

Salmacis sphaeroides (Linnaeus, 1758)

Salmacis sp. (incomplete)

Temnopleurus decipiens (de Meijere, 1904)

Temnotrema siamense (Mortensen, 1904)

Family Toxopneustidae

Nudechinus darnleyensis (Tenison-Woods, 1878)

Nudechinus scotiopremnus H.L. Clark, 1912

Tripneustes gratilla (Linnaeus, 1758)

Family Echinometridae

Echinostrephus molaris (Blainville, 1825)

Family Laganidae

Peronella lesueuri (Valenciennes, 1841)

Peronella orbicularis (Leske, 1778)

Peronella sp. (unidentifiable)

Family Echinolampadidae

Echinolampas ovata (Leske, 1778)

Family Schizasteridae

Schizaster sp. cf. compactus (Koehler, 1914)

Family Loveniidae

Breynia desorii Gray, 1851

Lovenia elongata (Gray, 1845)

\section{Class HOLOTHUROIDEA}

\section{Family Holothuriidae}

Actinopyga echinites (Jaeger, 1833)

Holothuria (Cystipus) inhabilis Selenka, 1867

Holothuria (Halodeima) atra Jaeger, 1833

Holothuria (Halodeima) edulis Lesson, 1830

Holothuria (Lessonothuria) pardalis Selenka, 1867

Holothuria (Mertensiothuria) leucospilota (Brandt, 1835)

Holothuria (Metriatyla) martensii Semper, 1868

Holothuria (Metriatyla) scabra Jaeger, 1833

Holothuria (Semperothuria) cinerascens (Brandt, 1835)

Holothuria (Stauropora) fuscocinerea Jaeger, 1833

Holothuria (Stauropora) pervicax Selenka, 1867

Holothuria (Thymiosycia) arenicola Semper, 1868

Holothuria (Thymiosycia) hilla Lesson, 1830

Holothuria (Thymiosycia) impatiens (Forskål, 1775)

Holothuria (Thymiosycia) sp.

Holothuria sp.

Family Stichopodidae

Stichopus chloronotus Brandt, 1835

Stichopus horrens Selenka, 1867

Stichopus sp. cf. horrens Selenka, 1867

Stichopus sp. cf. noctivagus Cherbonnier, 1980

\section{Family Cucumariidae}

Plesiocolochinus australis (Ludwig, 1875)

Pseudocolochirus violaceus (Théel, 1886)
$25 \mathrm{v}$

7

$23 \mathrm{~d}, 25 \mathrm{~d}$

9

$3 t, 8 t, v, 12 t, v, 13 t$, $\mathrm{v}, 16 \mathrm{v}, 22 \mathrm{v}$ $12 t, v, 18 t, v, 6 t, v$, $19 t, v, 22 t, v, 29 t, v$

$4 t, 15 t, v, 21 t, v, 26 t$, $\mathrm{v}, 27 \mathrm{t}, \mathrm{v}, 32 \mathrm{t}, \mathrm{v}$

$23 \mathrm{~d}$

$25 \mathrm{~d}$

$10,14 \mathrm{v}$

\section{$20,23 \mathrm{~d}$}

$11 \mathrm{~d}, 14 \mathrm{~d}, 20 \mathrm{~d}, 23 \mathrm{~d}, \quad 6$

25d, 28d, 31d

8

$14 \mathrm{~d}, 25 \mathrm{~d}$

23d

2d, 23d, 28d, 31d, 34d 19

11

$\begin{array}{lll}25 & 3,6 \mathrm{t}, \mathrm{v} & 4 \mathrm{v} \\ 31 & & \\ 7 \mathrm{v}, 10 \mathrm{v}, 11 \mathrm{v}, 14 \mathrm{v}, & 3 \mathrm{v}, 6 \mathrm{t}, \mathrm{v}, 9,12 \mathrm{t}, \mathrm{v}, & 4 \mathrm{v}, 15 \mathrm{t}, \mathrm{v}, 26 \mathrm{t}, 32 \mathrm{v} \\ 23 \mathrm{v}, 25 & 16 \mathrm{t}, \mathrm{v}, 19 \mathrm{t}, \mathrm{v}, 22,35 \mathrm{v} & \\ 17 & 3 \mathrm{v}, 8 \mathrm{v}, 13 \mathrm{v}, 16,18 \mathrm{t}, & 4,15 \mathrm{t}, \mathrm{v}, 26 \mathrm{t}, \mathrm{v}, 27 \mathrm{t}, \\ & \mathrm{v}, 19 \mathrm{t}, \mathrm{v} & \\ 14,28 & 82 \mathrm{v} \\ 7,10 \mathrm{v}, 11 \mathrm{v}, 14 \mathrm{v}, & 3,6 \mathrm{t}, \mathrm{v}, 9,12 \mathrm{v}, 16 & 32 \mathrm{v} \\ 25 \mathrm{v}, 34 \mathrm{v} & \\ 11 & & \\ 2,11,23 & 16,22 \\ 7,14 & 6 \mathrm{v}, 12 \mathrm{t}, \mathrm{v}, 16 \mathrm{v} \\ & \\ & 19 \\ 25 \mathrm{v} & 19 \mathrm{t}, 22 \mathrm{v} \\ & 9 \\ 14,28 \mathrm{v} & 19 \mathrm{t}, \mathrm{v}, 22 \mathrm{v} \\ 10,23,25 \mathrm{v}, 28 \mathrm{v} & 19 \mathrm{t}, 35 \mathrm{t}, \mathrm{v} \\ & 19 \\ & \end{array}$

$10 \mathrm{v}, 11 \mathrm{v}$

$23,31 \mathrm{v}$

$3 \mathrm{t}, 6 \mathrm{v}, 16 \mathrm{t}, \mathrm{v}, 35 \mathrm{t}, \mathrm{v}$ $32 t, v$
$4 \mathrm{v}, 15 \mathrm{t}, \mathrm{v}, 26 \mathrm{t}$ $\mathrm{v}, 32 \mathrm{t}, \mathrm{v}$
30

Depth $>10 \mathrm{~m}$ 


\section{Family Phyllophoridae}

Neothyonidium magnum (Ludwig, 1882)

Phyllophorus (Urodemella) brocki Ludwig, 1888

Family Sclerodactylidae

Afrocucumis africana (Semper, 1868) 
Table 2 Echinoderms from the Dampier Archipelago collected by the first diving expedition (DA1/98); species collected or observed at each station. $(v=$ visual or photographic record; $d=$ dead specimen; juv = juvenile).

\begin{tabular}{l} 
Station No. \\
\hline DAI/98/01 \\
Dolphin I., N tip \\
Dive \\
Max. depth $5.9 \mathrm{~m}$
\end{tabular}

Max. depth $5.9 \mathrm{~m}$

\section{DA1/98/02}

Dolphin I., E side

Intertidal

\section{DA1/98/03 \\ Legendre I., N side Dive \\ Max. depth $10.3 \mathrm{~m}$}

Inshore

\section{DA1/98/04}

Legendre I., $\mathrm{N}$ side Dive

Max. depth $11.7 \mathrm{~m}$

\section{DA1/98/05}

Legendre I., E tip Intertidal

\section{Species}

Nos in transect

Diadema setosum

Neothyonidium magnum

Comanthinasp.

Amphiura bidentata

Amphiura velox

Ophiactis savignyi

Ophiothrix ciliaris

Ophiothrix sp.

Ophiocomella sexradiata

Ophiomastix mixta

Ophiomastix variabilis

\section{Breynia desorii \\ Holothuria (Metriatyla) scabra}

No transect 1

Astropecten sumbawanus

Amphiura velox

Ophiactis fuscolineata

Ophiactis savignyi

Ophiactis sp.

Echinostrephus molaris

Stichopus chloronotus

Actinopyga echinites

Holothuria (Mertensiothuria) leucospilota

Linckia multifora

Amphioplus (Amphichilus) ochroleuca

Amphiura diacritica

Amphiura velox

Macrophiothrix 'caenosa'

Macrophiothrix lorioli

Macrophiothrix sp. juv.

Ophiactis savignyi

Ophiothrix ciliaris

Ophiothrix exigua

Culcita schmideliana

Diadema setosum

Holothuria (Halodeima) atra

Holothuda (Halodeima) edulis

Holothuria (Mertensiothuria) leucospilota

Clarkcomanthus cf. littoralis

Iconaster longimanus

Culcita schmideliana

Linckia multifora

Acanthaster planci

Amphiura velox

Dougaloplus echinatus

Ophiactis savignyi

Ophiothrix ciliaris

Ophioplocus imbricatus

Diadema setosum

Echinostrephus molaris

Actinopyga echinites

Holothuria (Halodeima) atra

Holothuria (Halodeima) edulis

Holothuria sp.

Ophiactis savignyi

Ophiothrix ciliaris

Ophiothrix exigua

$\begin{array}{cc}15 & 0 \\ 2 & 0 \\ 0 & 1 \\ 0 & 1 \\ 1 & 0 \\ & ?\end{array}$

1

0

0

$\begin{array}{ll}1 & 0 \\ 0 & 1 \\ 0 & 1 \\ 0 & 3 \\ 0 & 1\end{array}$

No transect
Nos outside transect
100 's in

mudflats

5 
Table 2 (cont.)

\begin{tabular}{|c|c|c|c|}
\hline Station No. & Species & $\begin{array}{c}\text { Nos in } \\
\text { transect }\end{array}$ & $\begin{array}{c}\text { Nos outside } \\
\text { transect }\end{array}$ \\
\hline
\end{tabular}

Ophiothrix plana

Synaptula recta

\section{DA1/98/06}

Haüy I., NW end Dive

Max. depth $3.1 \mathrm{~m}$

\section{DA1/98/07}

Gidley I., W side Intertidal

\section{DA1/98/08}

Angel I., W side Dive

Max. depth $9.1 \mathrm{~m}$

DA1/98/09

Angel I., W side

Dive

Max. depth $5.0 \mathrm{~m}$

\section{DA1/98/10}

Channel between Angel \& Gidley Is
Amphiura duncani

Ophiactis luteomaculata

Ophiactis savignyi

Macrophiothrix megapoma

Macrophiothrix sp. juv.

Ophiothrix ciliaris

Ophiothrix exigua

Ophiothrix plana

Ophionereis semoni

Ophioplocus imbricatus

Diadema setosum

0

Peronella orbicularis

Actinopyga echinites

Holothuria (Halodeima) atra

Holothuria (Mertensiothuria) leucospilota

Holothuria (Semperothuria) cinerascens

Stichopus chloronotus

Gymnanthenea globigera

Ophiactis fuscolineata

Ophiothrix ciliaris

Ophiothrix exigua

Ophiothrix plana

Salmacis sphaeroides

Holothuria (Halodeima) atra

Holothuria (Semperothuria) cinerascens

Holothuria (Mertensiothuria) leucospilota

1

0

0

0

1

1

No transect

1

1

3

1

Amphizra sp.

Ophiactis luteomaculata

Ophiactis savignyi

Macrophiothrix callizona

Macrophiothrix sp.

Ophiothela danae

Ophiothrix ciliaris

Ophiothrix plana

Ophiarachnella gorgonia

Ophiarachnella infernalis

Ophiodyscrita acosmeta

Ophioplocus imbricatus

Echinostrephus molaris

Peronella sp.

Diadema setosum

Holothuria edulis

Holothuria (Lessonothuria) pardalis

Holothuria (Thymiosycia) arenicola

Afrocucumis africana

Ophiactis luteomaculata

Macrophiothrix paucispina

Ophiothrix ciliaris

Temnopleurus decipiens

Holothuria (Halodeima) atra

Holothuria (Mertensiothuria) leucospilota

Holothuria (Thymiosycia) arenicola

Lamprometra palmata

Comanthina variabilis

No transect

Oxycomanthus sp.

Culcita schmideliana

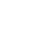


Station No.

Species
Nos in

transect
Nos outside transect
Intertidal

DA1/98/11

Dolphin I., W side Intertidal

\section{DA1/98/12}

Angel I., W side

Dive

Max. depth 6.6m

\section{DA1/98/13}

Hammersley Shoal

Dive

Max. depth $16.9 \mathrm{~m}$

\section{DA1/98/14}

Channel between Unnamed \& Gidley Is
Ophiactis luteomaculata

Ophiactis modesta

Ophiactis savignyi

Macrophiothrix 'caenosa'

Macrophiothrix paucispina

Ophiothrix (Keystonea) martensi

Ophiothrix (Keystonea) smaragdina

Ophiothrix ciliaris

Ophiothrix exigua

Ophiothrix plana

Ophiothrix sp.

Ophiocomella sexradiata

Ophionereis dubia

Tripneustes gratilla

Holothuria (Halodeima) atra

Holothuria (Mertensiothuria) leucospilota

Holothuria.(Thymiosycia) impatiens

Stichopus chloronotus

Luidia maculata

Pseudoreaster obtusangulus

Amphiura sp.

Macrophiothrix paucispina

Ophiothrix plana

Peronella orbicularis

Lovenia elongata

Holothuria (Halodeima) atra

Holothuria (Mertensiothuria) leucospilota

Holothuria (Metriatyla) martensii

Holothuria (Metriatyla) scabra

Stichopus chloronotus

Dorometra paroicirra

Ophiactis luteomaculata

Ophiactis savignyi

Macrophiothrix 'caenosa'

Ophiothrix (Keystonea) martensi

Ophiothrix ciliaris

Ophiothrix exigua

Ophiothrix plana

Ophiodyscrita acosmeta

Diadema setosum

Echinostrephus molaris

Holothuria (Halodeima) atra

Holothuria (Mertensiothuria) leucospilota

Holothuria (Semperothuria) cinerascens

Stichopus horrens

Stolus conjungens

Synaptula recta

Clarkcomanthus cf. littoralis

Amphiura velox

Ophiactis luteomaculata

Ophiactis sp. cf. modesta

Ophiactis savignyi

Ophiothela danae

Ophiothrix ciliaris

Ophiothrix plana

Phyllacanthus longispinus

Echinostrephus molaris

Holothuria (Halodeima) edulis

Culcita schmideliana

Pseudoreaster obtusangulus

Macrophiothrix paucispina
1

1

$<5$

1

$<4$

No transect

2

1

$2 ?$

1

2 ?

several

1

several

several

1

1

several

0

1

1

0

1

1

0

0

0

1

0

0

0

0 
Table 2 (cont.)

\begin{tabular}{|c|c|c|c|}
\hline Station No. & Species & $\begin{array}{c}\text { Nos in } \\
\text { transect }\end{array}$ & $\begin{array}{c}\text { Nos outside } \\
\text { transect }\end{array}$ \\
\hline Intertidal & $\begin{array}{l}\text { Ophiocoma dentata } \\
\text { Ophionereis dubia } \\
\text { Ophioplocus imbricatus } \\
\text { Tripneustes gratilla } \\
\text { Peronella orbicularis } \\
\text { Echinolampas ovata } \\
\text { Holothuria (Halodeima) atra } \\
\text { Holothuria (Lessonthuria) pardalis } \\
\text { Holothuria (Mertensiothuria) leucospilota } \\
\text { Holothuria (Semperothuria) cinerascens } \\
\text { Holothuria (Thymiosycia) hilla }\end{array}$ & & $\begin{array}{l}1 \\
4 \\
2 \\
1 \\
3 \\
5 \text { parts } \\
\text { many } \\
1 \\
\text { many } \\
\text { many } \\
\text { many }\end{array}$ \\
\hline $\begin{array}{l}\text { DA1/98/15 } \\
\text { Legendre I., NW end } \\
\text { Dive } \\
\text { Max. depth } 17.5 \mathrm{~m}\end{array}$ & $\begin{array}{l}\text { Oligometra carpenteri } \\
\text { Oligometra serripinna } \\
\text { Fromia sp. } \\
\text { Gomophia sphenisci } \\
\text { Linckia multifora } \\
\text { Ophiactis savignyi } \\
\text { Ophiothela danae } \\
\text { Ophiothrix (Keystonea) martensi } \\
\text { Ophiothrix (Keystonea) smaragdina } \\
\text { Ophiothrix ciliaris } \\
\text { Ophiothrix plana } \\
\text { Diadema setosum } \\
\text { Echinostrephus molaris } \\
\text { Holothuria (Halodeima) atra } \\
\text { Holothuria (Halodeima) edulis } \\
\text { Pseudocolochirus violaceus }\end{array}$ & $\begin{array}{c}60 \\
3 \\
3 \\
8 \\
0\end{array}$ & $\begin{array}{l}0 \\
0 \\
0 \\
0 \\
1\end{array}$ \\
\hline $\begin{array}{l}\text { DA1/98/16 } \\
\text { Hammersley Shoal } \\
\text { Dive } \\
\text { Max. depth } 3.4 \mathrm{~m}\end{array}$ & $\begin{array}{l}\text { Lamprometra palmata } \\
\text { Stephanometra indica } \\
\text { Culcita schmideliana } \\
\text { Gymnanthenea globigera } \\
\text { Acanthaster planci } \\
\text { Ophiactis luteomaculata } \\
\text { Ophiactis savignyi } \\
\text { Ophiothrix plana } \\
\text { Ophiocoma dentata } \\
\text { Ophiocoma sp. cf. pusilla } \\
\text { Ophiarachnella sphenisci } \\
\text { Ophioplocus imbricatus } \\
\text { Diadema setosum } \\
\text { Echinostrephus molaris } \\
\text { Holothuria (Halodeima) atra } \\
\text { Holothuria (Halodeima) edulis } \\
\text { Holothuria (Mertensiothuria) leucospilota } \\
\text { Holothuria (Metriatyla) scabra } \\
\text { Holothuria (Semperothuria) cinerascens } \\
\text { Stichopus chloronotus } \\
\text { Neothyonidium magnum }\end{array}$ & $\begin{array}{c}0 \\
0 \\
25 \\
0 \\
3 \\
0 \\
3 \\
0 \\
? \\
1 \\
2\end{array}$ & $\begin{array}{c}1 \\
4 \\
0 \\
3 \\
\text { several } \\
\text { several } \\
\text { several } \\
1 \\
? \\
0 \\
0\end{array}$ \\
\hline $\begin{array}{l}\text { DA1/98/17 } \\
\text { Wilcox I. } \\
\text { Intertidal }\end{array}$ & $\begin{array}{l}\text { Culcita schmideliana } \\
\text { Echinaster superbus } \\
\text { Ophiactis fuscolineata } \\
\text { Ophiactis savignyi } \\
\text { Ophiothrix ciliaris } \\
\text { Ophiothrix plana } \\
\text { Ophiarachnella sp. } \\
\text { Holothuria (Halodeima) edulis }\end{array}$ & No transect & $\begin{array}{c}1 \\
1 \\
4 \\
\text { several } \\
\text { several }\end{array}$ \\
\hline $\begin{array}{l}\text { DA1/98/18 } \\
\text { Haüy I. } \\
\text { Dive } \\
\text { Max. depth } 12.5 \mathrm{~m}\end{array}$ & $\begin{array}{l}\text { Acanthaster planci } \\
\text { Diadema setosum } \\
\text { Ophiarachnella sp. } \\
\text { Holothuria (Halodeima) edulis }\end{array}$ & $\begin{array}{c}1 \\
28 \\
2 \\
1\end{array}$ & $\begin{array}{l}0 \\
0 \\
0 \\
0\end{array}$ \\
\hline
\end{tabular}


Station No.

Species

Nos in

transect

Nos outside transect

DA1/98/19

Haüy I.

Dive

Max. depth $2.5 \mathrm{~m}$

Macrophiothrix paucispina

Ophiothrix ciliaris

0

1

Ophiothrix exigua

Ophiothrix plana

Ophiarachnella sp.

Diadema setosum

Breynia desorii

Holothuria (Halodeima) atra

Holothuria (Halodeima) edulis

Holothuria (Stauropora) fuscocinerea

Holothuria (Stautropora) pervicax

Holothuria (Thymiosycia) hilla

Holothuria (Thymiosycia) impatiens

Holothuria (Thymiosycia) sp.

\section{DA1/98/20 \\ Collier Rocks \\ Intertidal}

\section{DA1/98/21}

Delambre I, NW point Dive

Max. depth $16.9 \mathrm{~m}$

\section{DA1/98/22}

Delambre I., W side Dive

Max. depth $5.6 \mathrm{~m}$

\section{DA1/98/23}

Dolphin I., E side Intertidal
Ophiactis savignyi

Ophiothrix plana

Ophiarachnella infernalis

Ophiarachnella sphenisci

Peronella lesueuri

Peronella orbicularis

Phyllophorus (Urodemella) brocki

Clarkcomanthus littoralis

Fromia indica

Linckia multifora

Macrophiothrix 'caenosa'

Ophiothrix ciliaris

Ophiarachnella infernalis

Diadema setosum

Stephanometra indica

Ophiactis macrolepidota

Ophiactis savignyi

Ophiactis sp. cf. savignyi

Ophiactis sp.

Macrophiothrix 'caenosa'

Ophiothrix ciliaris

Ophiothrix sp. cf. ciliaris

Ophiothrix plana

Ophiomastix mixta

Ophionereis intermedia

Ophiarachnella gorgonia

Ophiolepis unicolor

Phyllacanthus longispinus

Diadema setosum

Echinostrephus molaris

Holothuria (Halodeima) atra

Holothuria (Metriatyla) scabra

Holothuria (Stauropora) pervicax

Holothuria (Thymiosycia) hilla

Stichopus noctivagus

Plesiocolochirus australis

Synaptula recta

Astropecten sumbawanus

Amphipholis squamata

Ophiactis fuscolineata

Ophiactis savignyi

Macrophiothrix 'caenosa'

Macrophiothrix megapoma

Macrophiothrix paucispina

Ophiothrix (Keystonea) martensi

Ophiothrix plana

Salmacis sp.

$\begin{array}{ll}0 & 1 \\ & 1 \\ & 1 \\ 5 & 1 \\ 1 & 0 \\ 0 & 0 \\ 1 & 1 \\ 1 & 0 \\ & \\ 5 & 0 \\ 6 & 0 \\ 2 & 0 \\ & \end{array}$

No transect

8

0

1

7

0

1

0

several several

1

1

several

2

1

1

No transect 
Table 2 (cont.)

\begin{tabular}{ll}
\hline Station No. & Species \\
\hline Nudechinus scotiopremnus \\
Peronella lesueuri \\
Peronella orbicularis \\
Breynia desorii \\
Schizaster sp. cf. compactus \\
Holothuria (Halodeima) atra \\
Holothuria (Metriatyla) sp. cf. scabra \\
Holothuria (Thymiosycia) impatiens \\
Stichopus horrens \\
Stichopus sp. cf. noctivagus \\
Holothuria fuscopunctata \\
Synaptula sp.
\end{tabular}

\section{DA1/98/24}

Dolphin I., E side Dive, Max. depth 5.4m

\section{DA1/98/25}

Keast I., S end Intertidal

DA1/98/26

Madeleine Shoals Dive

Max. depth $24.0 \mathrm{~m}$

\section{DA1/98/27}

Legendre I., $\mathrm{N}$ side Dive

Max. depth $16.2 \mathrm{~m}$
Neothyonidium magnum

Stichopus horrens

Comanthina variabilis

Stephanometra indica

Culcita schmideliana

Ophiactis savignyi

Macrophiothrix callizona

Ophiothrix ciliaris

Ophiothrix exigua

Ophiothrix plana

Diadema setosum

Salmacis sp.

Nudechinus scotiopremnus

Peronella orbicularis

Echinolampas ovata

Actinopyga echinites

Holothuria (Halodeima) atra

Holothuria (Mertensiothuria) leucospilota

Holothuria (Stauropora) pervicax

Holothuria (Thymiosycia) impatiens

Stephanometra oxyacantha

Oligometra carpenteri

Fromia indica

Gomophia sphenisci

Linckia multifora

Acanthaster planci

Echinaster varicolor

Ophiactis savignyi

Ophiothrix ciliaris

Ophiothrix plana

Diadema setosum

Echinostrephus molaris

Holothuria (Halodeima) atra

Holothuria (Halodeima) edulis

Colobometra perspinosa

Oligometra carpenteri

Culcita schmideliana

Fromia indica

Linckia multifora

Ophiactis sp. cf. modesta

Ophiactis savignyi

Ophiothela danae

Ophiothrix (Keystonea) martensi

Ophiothirx ciliaris

Ophiothrix exigua

\section{Nos in}

transect

$\begin{gathered}\text { Nos outside } \\ \text { transect }\end{gathered}$
2
3
15

3 \& parts

parts

1

1

I

!

many

2

0

0

No transect

1

1

several

2

2

several

parts

1

few

1

few

few

0

2

1

6

29

1

1

1

0

0

0

0

0

0

2

120

4 0

Half tranșect

0

$\begin{array}{ll}2 & 0 \\ 1 & 0 \\ 0 & 1 \\ 1 & 0\end{array}$


Ophiothrix plana

Diadema setosum

Holothuria (Halodeima) edulis

Neothyonidium magnum

\section{DA1/98/28}

Dolphin I., NW tip Intertidal

\section{DA1/98/29}

Legendre I., S side

Dive

Max. depth 5.0m

DA1/98/30

Burrup Peninsula, NE tip

Dive

Max. depth $10.9 \mathrm{~m}$

DA1/98/31

Searipple Passage, E end Intertidal

\section{DA1/98/32}

Legendre I., $\mathrm{N}$ side

Dive

Max. depth $15.9 \mathrm{~m}$

Ophiactis modesta

Peronella orbicularis

Breynia desorii

Holothuria (Thymiosycia) hilla

Comanthus wahlbergi

Comatula purpurea

Dorometra parvicirra

Amphipholis squamata

Ophiocentrus dilatata

Ophiactis luteomaculata

Ophiactis savignyi

Ophiothrix ciliaris

Ophiothrix plana

Ophiomastix variabilis

Phyllacanthus longispinus

Diadema setosum

Stichopus sp. cf. horrens

Zygometra punctata

Ophiomaza cacaotica

Phyllacanthus longispinus

Temnotrema siamense

Comanthus paroicirrus

Asterina sp. nov.

Ophiactis savignyi

Ophiactis sp. cf. savignyi

Macrophiothrix 'caenosa'

Macrophiothrix callizona

Macrophiothrix lineocaerulea

Macrophiothrix lorioli

Ophiothela danae

Ophiothrix ciliaris

Ophiothrix plana

Peronella orbicularis

Breynia desorii

Stichopus horrens

Stichopus noctivagus

Culcita schmideliana

Fromia indica

Acanthaster planci

Amphipholis squamata
Holothuria (Lessonothuria) pardalis.

Holothuria (Thymiosycia) impatiens

Phyllophorus (Urodemella) brocki

Ophiothrix (Keystonea) martensi

Phyllophorus (Urodemella) proteus

$\begin{array}{cc}10 & 0 \\ 1 & 0 \\ 0 & 1\end{array}$

No transect

several

1

1

2

3

1

1

2

2

several

\section{0}

1

1.

.

Ophiactis fuscolineata

Ophiactis sp. cf. modesta

Ophiactis savignyi

Macrophiothrix sp. juv.

Ophiothrix sp. cf. ciliaris

Diadema setosum

Echinostrephus molaris

Holothuria (Halodeima) atra

Holothuria (Halodeima) edulis

Holothuria (Mertensiothuria) leucospilota

Stichopus chloronotus

Neothyonidium magnum

1

several

several

3 in parts

2 \& parts

1

1 damaged

1

0

0

1 
Table 2 (cont.)

\begin{tabular}{|c|c|c|c|}
\hline Station No. & Species & $\begin{array}{l}\text { Nos in } \\
\text { transect }\end{array}$ & $\begin{array}{c}\text { Nos outside } \\
\text { transect }\end{array}$ \\
\hline $\begin{array}{l}\text { DA1/98/33 } \\
\text { Angel I., NE tip } \\
\text { Dive } \\
\text { Max. depth } 8.8 \mathrm{~m}\end{array}$ & $\begin{array}{l}\text { Comanthina variabilis } \\
\text { Oligometra carpenteri } \\
\text { Petasometra helianthoides } \\
\text { Amphioplus didymus } \\
\text { Amphiurd duncani } \\
\text { Amphiura velox } \\
\text { Ophiocentrus dilatata } \\
\text { Ophiocentrus sp. aff. dilatata } \\
\text { Ophiactis modesta } \\
\text { Ophiactis savignyi } \\
\text { Ophiothrix ciliaris } \\
\text { Ophiothrix plana } \\
\text { Ophiodyscrita acosmeta } \\
\text { Culcita schmideliana } \\
\text { Holothuria (Halodeima) atra } \\
\text { Stichopus horrens } \\
\text { Phyllophorus (Urodemella) brocki }\end{array}$ & $\begin{array}{l}1 \\
0 \\
1 \\
1\end{array}$ & $\begin{array}{l}0 \\
1 \\
0 \\
0\end{array}$ \\
\hline $\begin{array}{l}\text { DA1/98/34 } \\
\text { Tozer I. } \\
\text { Intertidal }\end{array}$ & $\begin{array}{l}\text { Ophiactis savignyi } \\
\text { Breynia desorii } \\
\text { Holothuria (Mertensiothuria) leucospilota }\end{array}$ & No transect & $\begin{array}{l}2 \\
1\end{array}$ \\
\hline $\begin{array}{l}\text { DA1/98/35 } \\
\text { Legendre I., S side } \\
\text { Snorkel }\end{array}$ & $\begin{array}{l}\text { Ophiactis savignyi } \\
\text { Macrophiothrix 'caenosa' } \\
\text { Holothuria (Halodeima) atra } \\
\text { Holothutria impatiens } \\
\text { Stichopus chloronotus } \\
\text { Stichopus horrens }\end{array}$ & $\begin{array}{l}0 \\
1 \\
4 \\
1\end{array}$ & $\begin{array}{l}1 \\
0 \\
0 \\
0\end{array}$ \\
\hline
\end{tabular}


Table 3 Echinoderms from the Dampier Archipelago collected by the second diving expedition (DA3/99); station numbers and depth ranges at which they were collected or observed within the transect or elsewhere. $(\mathrm{a}=$ from algae; $\mathrm{e}=$ from echinoderms; $\mathrm{s}=$ from sponge; $\mathrm{c}=$ from coral; $\mathrm{m}=$ from mollusc; $\mathrm{t}=$ collected on transect; $\mathrm{d}=$ dead test or fragments; $\mathrm{r}=$ from rock crevice; $\mathrm{v}=$ visual or photographic record).

\begin{tabular}{llll}
\hline & Intertidal & Depth 0-10 m & Depth 11-20 m \\
\hline ECHINODERMATA &
\end{tabular}

\section{Class CRINOIDEA}

Family Comasteridae

Clarkcomanthus littoralis (Carpenter, 1888)

Comanthina variabilis (Bell, 1882)

Comanthus alternans (Carpenter, 1881)

C. briareus (Bell, 1882)

C. gisleni Rowe et al. 1986

C. parvicirrus (Müller, 1841)

C. wahlbergii (Müller, 1843)

Comatella maculata (Carpenter, 1888)

C. stelligera (Carpenter, 1888)

Comatula pectinata (Linnaeus, 1758)

C. purpurea (Müller, 1843)

Family Zygometridae

Zygometra comata A.H. Clark, 1911

Z. microdiscus (Bell, 1882)

Z. punctata A.H. Clark, 1912

Family Himerometridae

Amphimetra tessellata (Müller, 1841)

Family Mariametridae

Lamprometra palmata (Müller, 1841)

Stephanometra indica (Smith, 1876)

S. spinipinna (Hartlaub, 1890)

Family Colobometridae

cf. Iconometra sp.

Oligometra carpenteri (Bell, 1884)

O. serripinna (Carpenter, 1881)

Family Antedonidae

Dorometra parvicirra (Carpenter, 1888)

\section{Class ASTEROIDEA}

Family Luidiidae

Luidia maculata Müller and Troschel, 1842

Family Astropectinidae

Astropecten granulatus Müller and Troschel, 184238

A. polyacanthus Müller and Troschel, 1842

A. sumbazuanus Döderlein, 1917

Family Goniasteridae

Iconaster longimanus (Möbius, 1859)

Styphlaster notabilis H.L. Clark, 1938

Family Oreasteridae

Anthenea conjungens Döderlein, 1935

Culcita schmideliana (Retzius, 1805)

Goniodiscaster acanthodes H.L. Clark, 1938

Gymnanthenea globigera (Döderlein, 1915)

Pentaceraster gracilis (Lütken, 1871)

Protoreaster lincki (Blainville, 1830)

P. nodulosus (Perrier, 1875)

Pseudoreaster obtusangulus (Lamarck, 1816)
48

51

38

$51,54,59 \mathrm{v}, 62 \mathrm{v}$

$36 \mathrm{t}, 47 \mathrm{t}$

$37 \mathrm{t}, 41,68 \mathrm{t}$

$52 t$

$67 t$

65

$37 \mathrm{t}, 49 \mathrm{t}$

$36 t, 37,61 t$

65

45

$\begin{array}{ll}36 \mathrm{t}, 47 \mathrm{t} & \\ 57 & \\ 68 & 55 \mathrm{t}, 65 \mathrm{t} \\ 49 \mathrm{t} & 65 \mathrm{t}\end{array}$

41

$55 t, 65 t$

$55 \mathrm{t}$

42

47

$40 \mathrm{t}, 46$

$40 \mathrm{t}, 68 \mathrm{t}$

$50 t$

$56 \mathrm{tv}$

$55 \mathrm{t}$

$52,55 t, 65$

65

47

$55 \mathrm{t}$

$55 \mathrm{t}$

57

45,48

$36,37 \mathrm{t}, 56 \mathrm{v}, 57 \mathrm{v}, 58 \mathrm{tv}$,

65 $61 \mathrm{v}, 68 \mathrm{v}$

45,48

$65 t$

$47 \mathrm{v}$

51

$45 \mathrm{v}, 48 \mathrm{v}, 51,54 \mathrm{v}$

61 tv

41

$55 \mathrm{v}$ 
Table 3 (cont.)

Family Ophidiasteridae
Fromia indica (Perrier, 1869)

Fromia sp. ?nov.

Gomophia sphenisci (A.M. Clark, 1967)

Hacelia helicosticha (Sladen, 1889)

Linckia laevigata (Linnaeus, 1758)

L. multifora (Lamarck, 1816)

Nardoa galatheae (Lütken, 1865)

Ophidiaster sp.

Ophidiaster sp. juv.

Tamaria tumescens (Koehler, 1910)

Tamaria sp.

Family Pterasteridae

Euretaster insignis (Sladen, 1882)

Family Asterinidae

Asterina anomala H.L. Clark, 1921

A. sarasini (de Loriol, 1897)

Family Acanthasteridae

Acanthaster planci (Linnaeus, 1758)

Family Echinasteridae

Echinaster superbus H.L. Clark, 1916

E. varicolor H.L. Clark, 1938

\section{Class OPHIUROIDEA}

\section{Family Amphiuridae}

Amphioplus (A.) stenaspis H.L. Clark, 1938

A. (Lymanella) depressa (Ljungman, 1867)

Amphipholis sp.

A. squamata (Delle-Chiaje, 1828)

Amphiura (A.) acrisia H.L. Clark, 1938

Amphiura (A.) constricta Lyman, 1879

A. (A.) duncani Lyman, 1882

Amphiura sp.

\section{Family Ophiactidae}

Ophiactis sp. cf. brachyura Döderlein, 1898

Ophiactis fuscolineata H.L. Clark, 1938

O. luteomaculata H.L. Clark, 1915

O. modesta Brock, 1888

Ophiactis savignyi (Müller and Troschel, 1842)

No locality

Intertidal

Depth 0-10 m

Depth 11-20 m

No locality

$\begin{array}{ll}57 \mathrm{t} & 52 \mathrm{t} \\ & 65 \mathrm{t} \\ & 52 \mathrm{t} \\ & 52 \\ 57 & 43 \\ 56 \mathrm{v} & 39 \mathrm{t}, 43 \mathrm{tv}, 52 \mathrm{v}, 67 \mathrm{tv} \\ \text { No locality } & 40 \mathrm{tv}, 46,50 \mathrm{tv}, 57 \mathrm{tv}, 67 \mathrm{v} \\ & 47 \mathrm{v}, \\ & 55 \mathrm{t} \\ & 55 \mathrm{t} \\ & 55\end{array}$

Near 48

45

64

$36 \mathrm{tv}, 40 \mathrm{v}, 44 \mathrm{v}, 46 \mathrm{v}, 49 \mathrm{v}, 50 \mathrm{v} \quad 43 \mathrm{v}, 52 \mathrm{tv}, 67 \mathrm{v}$

No locality

$56 t, 57$

$54 \mathrm{r}$
51
$59 \mathrm{r}$
$6 \mathrm{~m}$
$59 \mathrm{r}, 62 \mathrm{r}$
$66 \mathrm{~m}$
$38 \mathrm{c}, 45,48$

$38,42,45$

42,48

M. callizona H.L. Clark, 1938

M. lineocaerulea (H.L. Clark, 1928)

M. megapoma H.L. Clark, 1938

M. paucispina Hoggett, 1991

M. variabilis (Duncan, 1887)

Macrophiothrix sp.

Ophiomaza cacaotica Lyman, 1871

Ophiopteron elegans Ludwig, 1888

Ophiothela danae Verrill, 1869

Ophiothrix (Keystonea) martensi Lyman, 1874

O. (K.) smaragdina Studer , 1882

Ophiothrix ciliaris (Lamarck, 1816)
42

$38,42,54$

48

48,51

$38 c, 48,51$
67 trs

$56 r$

53 ts, 61 trs

$49 \mathrm{r}$

$40 \mathrm{tr}, 41 \mathrm{r}, 53 \mathrm{tr}$

70 ts

65 ts

65 trs

61 trs

47

$37 \mathrm{ts}, 46,49 \mathrm{t}, 53 \mathrm{trs}$,

$52 \operatorname{tr}$

$52 \mathrm{tr}, 55 \mathrm{~s}, 65 \mathrm{tr}, 67 \mathrm{tcrs}$ 67 trs

$56 \mathrm{ts}, 60 \mathrm{ts}, 61 \mathrm{trs}$

$37 \mathrm{tr}, 40 \mathrm{ts}, 58 \mathrm{ts}$

$65 \operatorname{tr}$

41,68 te

$37 t, 40 t, 47,49 t, 64 t$

65 tr

41 trs, 61 trs

68 te

61 trs

68te

$44 \mathrm{a}$

41 tg

$41 \mathrm{~s}, 46,49 \mathrm{t}, 53 \mathrm{tr}, 68 \mathrm{te}$

$56 \mathrm{ts}, 57 \mathrm{ts}$

$43 \mathrm{~m}, 65 \mathrm{tr}$

65 ts

$39 \mathrm{~s}, 55 \mathrm{ts}, 65 \mathrm{ts}$

$36 \mathrm{trs}, 37 \mathrm{trs}, 40 \mathrm{ts}, 41 \mathrm{~s}, \quad 55 \mathrm{ts}, 65 \mathrm{ts}, 67 \mathrm{ts}$

$47,53 \mathrm{ts}, 56 \mathrm{ts}, 58 \mathrm{ts}, 60 \mathrm{ts}$,

61 trs, 70 ts 


\begin{tabular}{llll} 
& Intertidal & Depth $\mathbf{0 - 1 0 ~ m ~}$ & Depth 11- \\
\hline O. exigua Lyman, 1874 & 42 & $40 \mathrm{ts}, 41 \mathrm{~s}, 56 \mathrm{ts}, 57 \mathrm{ts}$, & $55 \mathrm{ts}, 65 \mathrm{ts}$ \\
& & $60 \mathrm{ts}, 70 \mathrm{ts}$ & \\
O. plana Lyman, 1874 & $51,54 \mathrm{r}$ & $36 \mathrm{ts}, 40 \mathrm{ts}, 46,53,64$ & $43 \mathrm{~m}, 52 \mathrm{tr}$ \\
Ophiothrix sp. & 38 & 47 &
\end{tabular}

Family Ophiocomidae

Ophiomastix mixta Lütken, 1869

$44 \operatorname{tr}$

Family Ophionereididae

Ophionereis dubia (Müller and Troschel, 1842)

$38 c, 42,45,48,66$

O. intermedia A.M. Clark, 1953

48

O. semoni (Döderlein, 1896)

\section{Family Ophiodermatidae}

Ophiarachnella gorgonia (Müller and Troschel, 1842) 45

O. infernalis (Müller and Troschel, 1842)

Ophioconis cincta Brock, 1888

O. cupida Koehler, 1905

\section{Family Ophiuridae}

Ophiolepis unicolor H.L. Clark, 1938

Ophioplocus imbricatus (Müller and Troschel, 1842)

$42,54 \mathrm{r}$

\section{Class ECHINOIDEA}

\section{Family Cidaridae}

Phyllacanthus imperialis (Lamarck, 1816)

P. longispinus Mortensen, 1918

Prionocidaris bispinosa (Lamarck, 1816)

Family Diadematidae

Diadema setosum (Leske, 1778)

Family Temnopleuridae

Salmacis sp. (incomplete)

Temnopleurus sp. (incomplete)

Family Echinometridae

Echinostrephus molaris (Blainville, 1825)

\section{Family Laganidae}

Peronella lesueuri (Valenciennes, 1841)

P. sp. cf. macroproctes Koehler, 1922

P. sp. cf. orbicularis (Leske, 1778)

P. tuberculata Mortensen, 1918

Peronella spp

Family Echinolampadidae

Echinolampas ovata (Leske, 1778)

Family Fibulariidae

Fibularia sp. cf. acuta Yoshiwara, 1898

F. oblonga Gray, 1851

\section{Family Schizasteridae}

Schizaster compactus (Koehler, 1914) juv.

\section{Family Brissidae}

Brissus latecarinatus (Leske, 1778)

Metalia sternalis (Lamarck, 1816)

Rhynobrissus hemiasteroides A. Agassiz, 1879

Family Loveniidae

Breynia desorii Gray, 1851

$62 d$

$59 d$

\section{$54 \mathrm{v}$}

\section{2,48}

$45,48,51$

\section{$44 \mathrm{tr}, 46,49 \mathrm{t}, 57 \mathrm{ts}$ \\ 47}

$37 \mathrm{tr}, 44 \mathrm{tr}, 47,49 \mathrm{t}$
49

47

$47,53 \mathrm{t}$

$37 \mathrm{tr}, 46,47,53 \mathrm{t}$

65 tr

65 tr

46

v, no locality

36d, 40d, 46d

$36 \mathrm{tv}, 44 \mathrm{tv}, 49 \mathrm{tv}, 53 \mathrm{tv}$, $57 \mathrm{tv}, 58 \mathrm{tv}, 60 \mathrm{tv}$, 63tv, 64tv

38d, 45d, 54d, 66d

$38 \mathrm{~d}, 51 \mathrm{~d}, 54 \mathrm{~d}$

$38 \mathrm{~d}, 54 \mathrm{~d}$

$38 \mathrm{~d}, 62 \mathrm{~d}$

$38 \mathrm{~d}, 42 \mathrm{~d}$

d, no locality

No locality

40 tv $, 44,46 \mathrm{tv}$

$43 \mathrm{tv}, 50 \mathrm{tv}, 52 \mathrm{tv}, 67 \mathrm{v}$

$41,56 \mathrm{tv}, 68 \mathrm{tv}$

$55 \mathrm{tv}$

$47 \mathrm{~d}, 61 \mathrm{~d}, 70 \mathrm{~d}$

$47 d$

41d, 68d

65 td, 69

65 td
$50 \mathrm{tv}, 52 \mathrm{tv}, 65 \mathrm{v}$ $67 \mathrm{tv}, 69 \mathrm{tv}$

\section{$37 \mathrm{td}$}

$49 d$

68

$41 d$

68

$38,42 d, 54 d$ 
Table 3 (cont.)

\begin{tabular}{|c|c|c|c|}
\hline & Intertidal & Depth 0-10 m & Depth 11-20 m \\
\hline \multicolumn{4}{|l|}{ Family Spatangidae } \\
\hline $\begin{array}{l}\text { Nacospatangus (Pseudomaretia) interruptus } \\
\quad \text { (Studer, 1880) }\end{array}$ & $42 \mathrm{~d}$ & $57,68 \mathrm{~d}$ & \\
\hline \multicolumn{4}{|l|}{ Class HOLOTHUROIDEA } \\
\hline Family Holothuriidae & & . & \\
\hline Actinopyga echinites (Jaeger, 1,833) & $42 \mathrm{v}, 45 \mathrm{v}, 48,66 \mathrm{v}$ & $37 \mathrm{tv}, 40,44 \mathrm{tv}, 47 \mathrm{tv}, 53 \mathrm{v}$ & \\
\hline Bohadschia marmorata (Jaeger, 1833) & & $56 \mathrm{t}$ & \\
\hline Holothuria (Halodeima) atra Jaeger, 1833 & $42,45 \mathrm{v}, 48 \mathrm{v}, 51 \mathrm{v}, 66 \mathrm{v}$ & $\begin{array}{l}44 \mathrm{v}, 49 \mathrm{tv}, 56 \mathrm{tv}, 57 \mathrm{tv} \\
64 \mathrm{tv}, 70 \mathrm{tv}\end{array}$ & \\
\hline H. (Halodeima) edulis Lesson, 1830 & $42 \mathrm{v}, 51 \mathrm{v}$ & $\begin{array}{l}36 \mathrm{tv}, 44 \mathrm{tv}, 47 \mathrm{tv}, 49 \mathrm{tv} \\
58 \mathrm{tv}, 64 \mathrm{tv}\end{array}$ & $50 \mathrm{tv}, 52 \mathrm{tv}, 67 \mathrm{tv}$ \\
\hline H. (Lessonothuria) pardalis Selenka, 1867 & $45,51 v, 59 v, 62$ & $\begin{array}{l}36,49 \mathrm{tv}, 53 \mathrm{tv}, 60 \mathrm{tv} \\
63 \mathrm{tv}, 64 \mathrm{tv}\end{array}$ & 67 \\
\hline H. (Lessonothuria) sp. juv. & $51,59,62$ & & \\
\hline \multicolumn{4}{|l|}{ Holothuria (Mertensiothuria) leucospilota } \\
\hline (Brandt, 1835) & $\begin{array}{l}42 \mathrm{v}, 45 \mathrm{v}, 48 \mathrm{v}, 51 \mathrm{v} \\
54 \mathrm{v}, 59 \mathrm{v}, 66 \mathrm{v}\end{array}$ & $\begin{array}{l}37 \mathrm{tv}, 47 \mathrm{tv}, 56 \mathrm{tv} \\
58 \mathrm{tv}, 64 \mathrm{tv}, 70 \mathrm{tv}\end{array}$ & $52 \mathrm{tv}$ \\
\hline H. (Metriatyla) scabra Jaeger, 1833 & $38 \mathrm{v}, 54$ & & \\
\hline H. (Platyperona) difficilis Semper, 1868 & 45 & & \\
\hline Holothuria (Semperothuria) cinerascens (Brandt, 1835) & $45,48 \mathrm{v}$ & & \\
\hline H. (Thymiosycia) hilla Lesson, 1830 & $42,45 \mathrm{v}, 62 \mathrm{v}$ & $47 \mathrm{v}, 49 \mathrm{tv}$ & \\
\hline H. (Thymiosycia) impatiens (Forskål, 1775) & $\begin{array}{l}38 \mathrm{v}, 42 \mathrm{v}, 45 \mathrm{v} \\
48 \mathrm{v}, 54 \mathrm{v}\end{array}$ & $44 t v$ & \\
\hline H. (Thymiosycia) sp. & 38 & & \\
\hline \multicolumn{4}{|l|}{ Family Stichopodidae } \\
\hline Stichopus chloronotus Brandt, 1835 & $42,45 v, 51$ & $44 t v, 56,58 \mathrm{tv}$ & \\
\hline S. hermanni Semper, 1868 & 54 & $57 \mathrm{v}$ & \\
\hline Stichopus sp. cf. horrens Selenka, 1867 & $42,45 \mathrm{v}, 51$ & $44 \mathrm{tv}, 56 \mathrm{tv}, 61 \mathrm{tv}, 64 \mathrm{tv}$ & \\
\hline $\begin{array}{l}\text { S. sp. cf. monotuberculatus Quoy and } \\
\text { Gaimard, } 1833\end{array}$ & & 41 & \\
\hline S. variegatus group & 42 & & \\
\hline Stichopus sp. & & 41,56 & \\
\hline \multicolumn{4}{|l|}{ Family Cucumariidae } \\
\hline Actinocucumis typica Ludwig, 1875 & 45 & & \\
\hline Cercodemas anceps Selenka, 1867 & & $68 \mathrm{t}$ & \\
\hline Colochirus crassus Ekman, 1918 & & 41 & \\
\hline Leptopentacta grisea H.L. Clark, 1938 & 45 & $47,70 t$ & \\
\hline Plesiocolochirus australis (Ludwig, 1875) & & 53 & \\
\hline Pseudocolochirus violaceus (Théel, 1886) & & $57 \mathrm{v}$ & $52 \mathrm{v}, 55 \mathrm{tv}, 65 \mathrm{tv}$ \\
\hline \multicolumn{4}{|l|}{ Family Phyllophoridae } \\
\hline Neothyonidium magnum (Ludwig, 1882) & & $37,61 \mathrm{tv}$ & \\
\hline Phyllophorus (Urodemella) proteus Bell, 1884 & 45 & & \\
\hline \multicolumn{4}{|l|}{ Family Sclerodactylidae } \\
\hline Cladolabes schmeltzii (Ludwig, 1875) & 45 & & \\
\hline \multicolumn{4}{|l|}{ Family Synaptidae } \\
\hline Synaptula macra H.L. Clark, 1938 & & $64 t$ & \\
\hline S. recta Semper, 1868 & 62 & $41,56 \mathrm{tv}, 68 \mathrm{tv}$ & $55 \mathrm{t}$ \\
\hline
\end{tabular}


Table 4 Echinoderms from the Dampier Archipelago collected by the second diving expedition (DA3/99); locality, depth, species and numbers collected or observed from each station. $(v=$ visual or photographic record; $d=$ dead specimen; juv = juvenile).

\begin{tabular}{lccc} 
Station No. Species & $\begin{array}{c}\text { Nos in } \\
\text { transect }\end{array}$ & $\begin{array}{c}\text { Nos outside } \\
\text { transect }\end{array}$ \\
\hline
\end{tabular}

DA3/99/36

N side of Malus I.

Dive, Coll: S. Morrison 27/8/99 8.5m

DA3/99/37

NW side of Malus I.

Dive, Coll: S. Morrison 27/8/99 3.5m

\section{DA3/99/38}

$\mathrm{N}$ side of Malus I. Intertidal

Coll: S. Morrison 27/8/99

\section{DA3/99/39}

NNE of Brigadier I.

Dive, Coll: S. Morrison 28/8/99 16m

\section{DA3/99/40}

W of Brigadier I.

Dive, Coll: S. Morrison 28/8/99 8m

\section{Clarkcomanthus littoralis}

Comatella maculata

Comanthus parvicirrus

Crinoid, black with yellow tips on pinnules

Culcita schmideliana

Acanthaster planci

Ophiothrix ciliaris

Ophiothrix plana

Phyllacanthus longispinus(d)

Diadema setosum

Holothuria edulis

Holothuria pardalis

Comanthus gisleni

Comanthina variabilis

Crinoid, yellow

Culcita schmideliana

Ophiactis savignyi

Ophiactis sp.

Ophiothrix ciliaris

Macrophiothrix 'caenosa'

Ophiarachnella gorgonia

Ophioplocus imbricatus

Breynia dessorii (d)

Echinolampas ovata (d)

Actinopyga echinites

Holothuria leucospilota

Neothyonidium magnum

Astropecten polyacanthus

Astropecten granulatus

Ophiactis savignyi

Macrophiothrix paucispina

Macrophiothrix 'caenosa'

Ophiothrix ciliaris

Ophionereis dubia

Breynia desorii

Echinolampas ovata (d)

Peronella tuberculata (d)

Peronella cf. orbicularis (d)

Peronella cf. macroproctes (d)

Peronella sp. (d)

Holothuria impatiens

Holothuria scabra

Holothuria sp.

Crinoid, yellow

Crinoid, black

Linckia multifora

Ophiothrix smaragdina

Phyllacanthus longispinus (d)

Stephanometra indica

Stephanometra spinipinna

Crinoid, yellow

Crinoid, black

Linckia multifora

Acanthaster planci

Amphiura duncani

Ophiactis sp.

Macrophiothrix 'caenosa'

1

2

2

1(v)

Several

$$
1(v)
$$

3

1

Several

$1(\mathrm{v})$

1(v)$$
1
$$

1 (v)

2(v)

7

2

1

$1(\mathrm{v})$

5(v)

2

1
Several<smiles>[AlH]</smiles>

1

1

1

1

6

12

1 (v)

Several(v)

Several

$$
\begin{gathered}
\text { Many } \\
1 \\
1 \\
2 \\
1(v) \\
\text { Several(v) } \\
2(v)
\end{gathered}
$$


Table 4 (cont.)

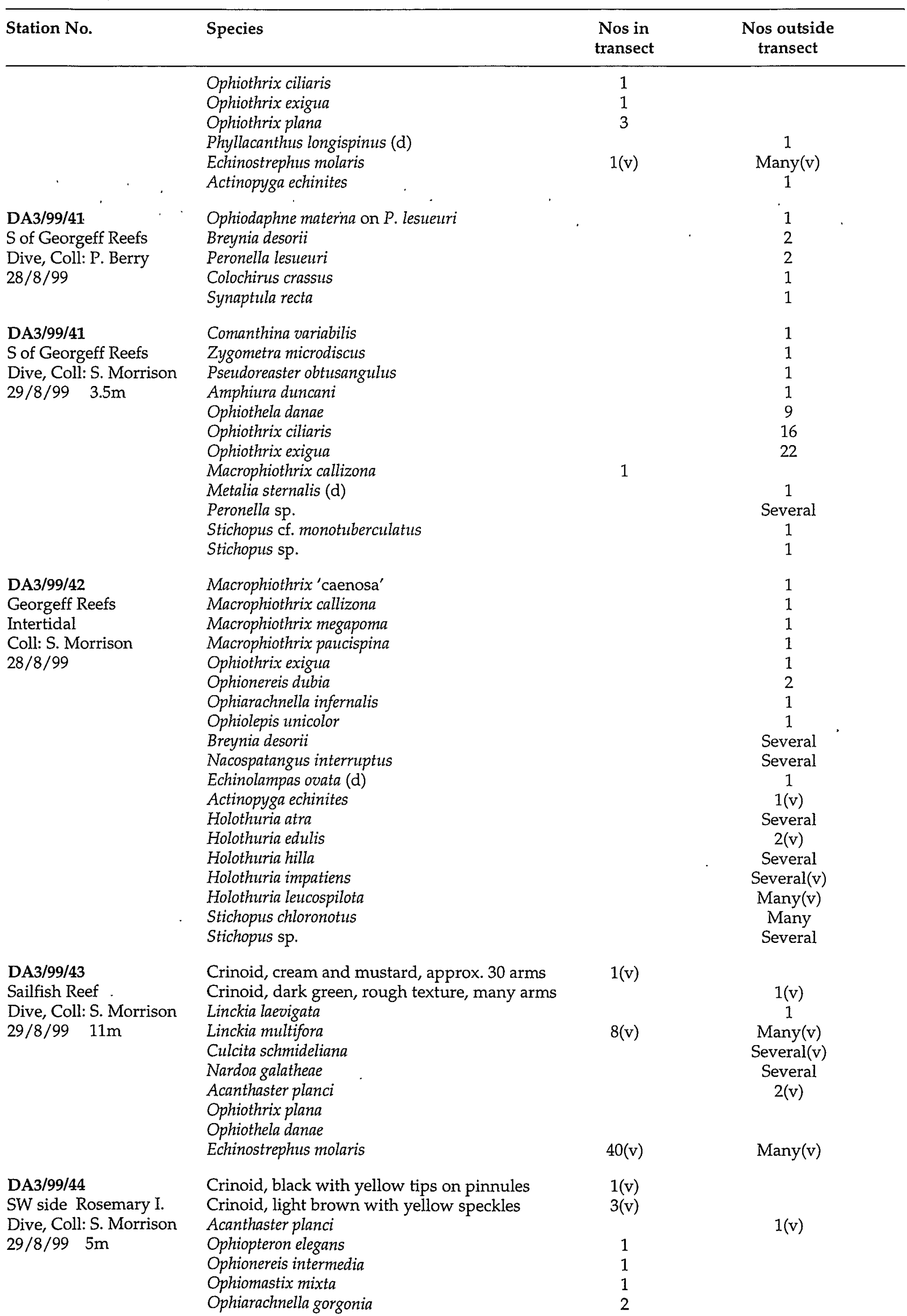


DA3/99/45

Tish Reef, Rosemary I. Intertidal

Coll: S. Morrison

$30 / 8 / 99$

\section{DA3/99/46}

NW Kendrew I.

Dive, Coll: S. Morrison 30/8/99 5m

\section{DA3/99/47}

SE Kendrew I.

Dive, Coll: S. Morrison 30/8/99 4m
Diadema setosum

Echinostrephus molaris

Actinopyga echinites

Holothuria edulis

Holothuria atra

Holothuria impatiens

Stichopus chloronotus

Stichopus horrens

Comanthus wahlbergi

Culcita schmidelaina juv.

Pseudoreaster obtusangulus

Gymnanthenea globigera

Asterina sarasini

Ophiactis savignyi

Macrophiothrix 'caenosa'

Ophionereis dubia

Ophiarachnella gorgonia

Ophioplocus imbricatus

Peronella cf. macroproctes (d)

Actinopyga echinites

Holothuria atra

Holothuria cinerascens

Holothuria difficilis

Holothuria hilla

Holothuria impatiens

Holothuria leucospilota

Holothuria pardalis

Stichopus chloronotus

Stichopus horrens

Cladolabes schmeltzii

Actinocucumis typica

Leptopentacta grisea

Stephanometra indica

Linckia multifora

Acanthaster planci

Ophiactis savignyi

Ophiothrix martensi

Ophiothrix plana

Ophionereis intermedia

Ophioplocus imbricatus

Phyllacanthus imperialis

Phyllacanthus longispinus (d)

Echinostrephus molaris

Comatella maculata

Clarkcomanthus littoralis

Lamprometra palmata

Crinoid, black and orange

Crinoid, black, many arms

Protoreaster lincki

Pentaceraster gracilis

Nardoa galatheae

Ophiactis cf. fuscolineata

Ophiothrix sp.

Macrophiothrix 'caenosa'

Ophiothrix ciliaris

Ophionereis semoni

Ophioconis cutpida

Ophioplocus imbricatus

Peronella cf. macroproctes (d)

Peronella cf. orbicularis (d)

Actinopyga echinites

Holothuria edulis

$7(\mathrm{v})$
$10(\mathrm{v})$
7
$1(\mathrm{v})$
$16(\mathrm{v})$
$5(\mathrm{v})$

Few

1

Many(v)

Several(v)

Few(v)

Many

$5(\mathrm{v})$

2

Few

Few(v)

Several

1

1

4

2

1

1

Many(v)

Several(v)

Many

Many

Many(v)

Many(v)

Several $(v)$

Many(v)

Few(v)

1

Several

Few

1

Many(v)

2(v)

9

1

1

1

1

1

1

5(v)

Many(v)

1

Several

Several

Several

1 (v)

$8(\mathrm{v})$

Several(v)

1

1

1

1

1

1

2

1

1

1

4

28(v)

1(v) 
Table 4 (cont.)

\begin{tabular}{|c|c|c|c|}
\hline Station No. & Species & $\begin{array}{l}\text { Nos in } \\
\text { transect }\end{array}$ & $\begin{array}{c}\text { Nos outside } \\
\text { transect }\end{array}$ \\
\hline & $\begin{array}{l}\text { Holothuria leucospilota } \\
\text { Holothuria hilla } \\
\text { Leptopentacta grisea }\end{array}$ & $1(v)$ & $\begin{array}{l}\text { Few } \\
1(\mathrm{v}) \\
1\end{array}$ \\
\hline $\begin{array}{l}30 / 8 / 99 \text { (not a station) } \\
\text { NE Goodwyn I. } \\
\text { Coll. C. Bryce }\end{array}$ & $\begin{array}{l}\text { Euretaster insignis } \\
\text { Gymnanthenea globigera }\end{array}$ & & $\begin{array}{l}1 \\
1\end{array}$ \\
\hline $\begin{array}{l}\text { DA3/99/48 } \\
\text { N Goodwyn I. } \\
\text { Intertidal } \\
\text { Coll: P. Morrison } \\
\text { 31/8/99 }\end{array}$ & $\begin{array}{l}\text { Clarkcomanthus littoralis } \\
\text { Crinoid, yellow and green, many hooked arms } \\
\text { Crinoid, small and brown, } 9 \text { arms } \\
\text { Culcita schmideliana } \\
\text { Gymnanthenea globigera } \\
\text { Pseudoreaster obtusangulus } \\
\text { Ophiactis savignyi } \\
\text { Macrophiothrix callizona } \\
\text { Macrophiothrix variabilis } \\
\text { Macrophiothrix sp. } \\
\text { Ophionereis dubia } \\
\text { Ophionereis intermedia } \\
\text { Diadema setosum } \\
\text { Actinopyga echinites } \\
\text { Holothuria atra } \\
\text { Holothuria cinerascens } \\
\text { Holothuria impatiens } \\
\text { Holothuria leucospilota }\end{array}$ & & $\begin{array}{c}1 \\
1(\mathrm{v}) \\
1(\mathrm{v}) \\
1 \\
1 \\
1(\mathrm{v}) \\
1 \\
2 \\
1 \\
1 \\
3 \\
1 \\
1(\mathrm{v}) \\
1 \\
1(\mathrm{v}) \\
1(\mathrm{v}) \\
1(\mathrm{v}) \\
1(\mathrm{v})\end{array}$ \\
\hline $\begin{array}{l}\text { DA3/99/49 } \\
\text { S Goodwyn I. } \\
\text { Dive, Coll: P. Morrison } \\
31 / 8 / 99 \quad 5 \mathrm{~m}\end{array}$ & $\begin{array}{l}\text { Comanthus gisleni } \\
\text { Comatula purpurea } \\
\text { Acanthaster planci } \\
\text { Ophiactis savignyi } \\
\text { Macrophiothrix 'caenosa' } \\
\text { Ophiothrix martensi } \\
\text { Ophionereis intermedia } \\
\text { Ophiarachnella gorgonia } \\
\text { Diadema setosum } \\
\text { Fibularia cf. acuta (d) } \\
\text { Holothutria atra } \\
\text { Holothuria edulis } \\
\text { Holothuria hilla } \\
\text { Holothuria pardalis }\end{array}$ & $\begin{array}{c}1 \\
1 \\
2 \\
2 \\
1 \\
3 \\
1 \\
92(\mathrm{v}) \\
1 \\
1(\mathrm{v}) \\
1(\mathrm{v}) \\
4(\mathrm{v}) \\
3(\mathrm{v})\end{array}$ & $1(v)$ \\
\hline $\begin{array}{l}\text { DA3/99/50 } \\
\text { E side of Bare Rock } \\
\text { Dive, Coll: P. Morrison } \\
31 / 8 / 99 \quad 15 \mathrm{~m}\end{array}$ & $\begin{array}{l}\text { Stephanometra indica } \\
\text { Crinoid, bright green and yellow } \\
\text { Linckia multifora } \\
\text { Acanthaster planci } \\
\text { Phyllacanthus longispinus } \\
\text { Diadema setosum } \\
\text { Echinostrephus molaris } \\
\text { Holothuria edulis }\end{array}$ & $\begin{array}{c}2 \\
1(\mathrm{v}) \\
10(\mathrm{v}) \\
\\
5(\mathrm{v}) \\
1(\mathrm{v}) \\
2(\mathrm{v}) \\
1(\mathrm{v})\end{array}$ & $1(\mathrm{v})$ \\
\hline $\begin{array}{l}\text { DA3/99/51 } \\
\text { N side Enderby I. } \\
\text { Intertidal } \\
\text { Coll: S. Morrison } \\
\text { 1/9/99 }\end{array}$ & $\begin{array}{l}\text { Luidia maculata } \\
\text { Astropecten sumbawanus } \\
\text { Protoreaster nodulosus } \\
\text { Pseudoreaster obtusangulus } \\
\text { Amphipholis sp. } \\
\text { Macrophiothrix sp. } \\
\text { Ophiothrix ciliaris } \\
\text { Ophiothrix plana } \\
\text { Ophioplocus imbricatus } \\
\text { Peronella cf. orbicularis (d) } \\
\text { Holothuria atra } \\
\text { Holothuria edulis } \\
\text { Holothuria leucospilota }\end{array}$ & & $\begin{array}{c}1 \\
1 \\
\text { Many } \\
\text { several } \\
1 \\
1(\mathrm{v}) \\
1 \\
1 \\
2 \\
5 \\
\text { Many(v) } \\
\text { Few(v) } \\
\text { Many(v) }\end{array}$ \\
\hline
\end{tabular}


Station No.

Species
Nos in

transect

Holothuria (Lessonothuria) sp. juv.

Holothuria pardalis

Stichopus chloronotus

Stichopus horrens

DA3/99/52

NW Roly Rock

Dive, Coll: R. Peart

$1 / 9 / 99 \quad 15 \mathrm{~m}$

DA3/99/53

NE Enderby I.

Dive, Coll: R. Peart

$1 / 9 / 995 \mathrm{~m}$

\section{DA3/99/54}

E Enderby I/

Intertidal

Coll: S. Morrison

2/9/99

transect

Clarkcomanthus littoralis

Oligometra carpenteri

Crinoid, yellow-green

Crinoid, black

Culcita schmideliana

Acanthaster planci

Fromia indica

Gomophia sphenisci

Linckia multifora

Hacelia helicosticha

Ophiactis luteomaculata

Ophiactis modesta

Ophiothrix plana

Diadema setosum

Echinostrephus molaris

Holothuria edulis

Holothuria leucospilota

Pseudocolchirus violaceus

Crinoid, black

Crinoid, black with yellow tips on pinnules

Amphipholis squamata

Amphiura duncani

Ophiactis savignyi

Ophiothrix ciliaris

Ophiothrix martensi

Ophiolepis unicolor

Ophioplocus imbricatus

Diadema setosum

Actinopyga echinites

Holothuria pardalis

Plesiocolochirus australis

Astropecten sumbawanus

Pseudoreaster obtusangulus

Amphioplus depressus

Macrophiothrix paucispina

Ophiothrix plana

Ophiarachnella infernalis

Peronella lesueuri

Peronella cf. macroproctes (d)

Peronella cf. orbicularis (d)

Peronella tuberculata (d)

Breynia desorii (d)

Holothuria scabra

Holothuria leucospilota

Holothuria impatiens

Stichopus hermanni

Comanthus alternans

Comatula pectinata

Zygometra comata

Zygometra punctata

Oligometra carpenteri

cf. Iconometra sp.

Amphimetra cf. tessellata

Iconaster longimanus

Tamaria tumescens

Styphlaster notabilis

Ophidiaster sp. juv.
Nos outside transect
Dive, Coll: S. Morrison

2/9/99 17m
1

Few

Few

1

3(v)

1

Several(v)

1(v)

1

1

13

3

57(v)

$45(\mathrm{v})$

4(v)

1 (v)

$1(v)$

$1(\mathrm{v})$
1 (v)
1
1
7
6
1
1
1
$101(\mathrm{v})$

$1(\mathrm{v})$

1

1
3

Several

10

Many

$>400$

4

32

3

1

1

1 
Table 4 (cont.)

\begin{tabular}{|c|c|c|c|}
\hline Station No. & Species & $\begin{array}{l}\text { Nos in } \\
\text { transect }\end{array}$ & $\begin{array}{c}\text { Nos outside } \\
\text { transect }\end{array}$ \\
\hline v & $\begin{array}{l}\text { Pentaceraster gracilis } \\
\text { Ophiothrix ciliaris } \\
\text { Ophiothrix exigua } \\
\text { Ophiothrix smaragdina } \\
\text { Peronella lesueuri } \\
\text { Pseudocolchirus violaceus } \\
\text { Synaptula recta }\end{array}$ & $\begin{array}{c}1(\mathrm{v}) \\
3 \\
17 \\
1 \\
1(\mathrm{v}) \\
11(\mathrm{v}) \\
4\end{array}$ & \\
\hline $\begin{array}{l}\text { DA3/99/56 } \\
\text { NW tip Eaglehawk I. } \\
\text { Dive Coll: R. Peart } \\
\begin{array}{l}3 / 9 / 99 \quad 9 \mathrm{~m}\end{array}\end{array}$ & $\begin{array}{l}\text { cf. Iconometra sp. } \\
\text { Culcita schmideliana } \\
\text { Linckia laevigata } \\
\text { Echinaster varicolor } \\
\text { Ophiactis savignyi } \\
\text { Ophiothrix ciliaris } \\
\text { Ophiothrix exigua } \\
\text { Ophiothrix smaragdina } \\
\text { Peronella lesueuri } \\
\text { Bohadschia marmorata } \\
\text { Holothuria atra } \\
\text { Holothuria leucospilota } \\
\text { Stichopus horrens } \\
\text { Stichopus sp. } \\
\text { Synaptula recta }\end{array}$ & $\begin{array}{c}1 \\
5 \\
2 \\
14 \\
2 \\
1(\mathrm{v}) \\
1 \\
1(\mathrm{v}) \\
1(\mathrm{v}) \\
1(\mathrm{v}) \\
2(\mathrm{v})\end{array}$ & $\begin{array}{l}1(\mathrm{v}) \\
\\
2(\mathrm{v}) \\
1 \\
\text { Many }\end{array}$ \\
\hline $\begin{array}{l}\text { DA3/99/57 } \\
\text { NE of NW Reefs } \\
\text { Dive, Coll: R. Peart } \\
3 / 9 / 999 \mathrm{~m}\end{array}$ & $\begin{array}{l}\text { Comatella stelligera } \\
\text { Culcita schmideliana } \\
\text { Fromia indica } \\
\text { Linckia multifora } \\
\text { Hacelia helicosticha } \\
\text { Anthenea conjungens } \\
\text { Ophiothrix exigua } \\
\text { Ophiothrix smaragdina } \\
\text { Ophionereis intermedia } \\
\text { Diadema setosum } \\
\text { Nacospatangus interruptus } \\
\text { Holothuria atra } \\
\text { Stichopus hermanni } \\
\text { Pseudocolchirus violaceus }\end{array}$ & $\begin{array}{c}3 \\
2 \\
5(\mathrm{v}) \\
\\
1 \\
1 \\
1 \\
26(\mathrm{v}) \\
2(\mathrm{v})\end{array}$ & $\begin{array}{c}1(\mathrm{v}) \\
2 \\
\\
\\
4 \\
1(\mathrm{v}) \\
1(\mathrm{v})\end{array}$ \\
\hline $\begin{array}{l}\text { DA3/99/58 } \\
\text { NE side Enderby I. } \\
\text { Dive, Coll: P. Morrison } \\
3 / 9 / 99 \quad 3.5 \mathrm{~m}\end{array}$ & $\begin{array}{l}\text { Culcita schmideliana } \\
\text { Ophiactis sp. } \\
\text { Ophiothrix ciliaris } \\
\text { Diadema setosum } \\
\text { Nacospatangus interruptus (d) } \\
\text { Holothuria edulis } \\
\text { Holothuria leucospilota } \\
\text { Stichopus chloronotus }\end{array}$ & $\begin{array}{l}1(\mathrm{v}) \\
1 \\
6 \\
10(\mathrm{v}) \\
3 \\
1(\mathrm{v}) \\
1(\mathrm{v}) \\
1(\mathrm{v})\end{array}$ & \\
\hline $\begin{array}{l}\text { DA3/99/59 } \\
\text { NW side W Lewis I. } \\
\text { Intertidal } \\
\text { Coll: S. Morrison } \\
\text { 4/9/99 }\end{array}$ & $\begin{array}{l}\text { Astropecten cf. sumbawanus } \\
\text { Amphipholis squamata } \\
\text { Ophiactis cf. fuscolineata } \\
\text { Temnopleurus sp. } \\
\text { Holothuria leucospilota } \\
\text { Holothuria pardalis } \\
\text { Holothuria (Lessonothuria) sp. (juv) }\end{array}$ & & $\begin{array}{c}1(\mathrm{v}) \\
2 \\
1 \\
1 \\
\text { Few }(\mathrm{v}) \\
\text { Several(v) } \\
1\end{array}$ \\
\hline $\begin{array}{l}\text { DA3/99/60 } \\
\text { N West Lewis I. } \\
\text { Dive, Coll: P. Morrison } \\
\begin{array}{ll}4 / 9 / 99 \quad 2.5 \mathrm{~m}\end{array}\end{array}$ & $\begin{array}{l}\text { Ophiactis savignyi } \\
\text { Ophiothrix ciliaris } \\
\text { Ophiothrix exigua } \\
\text { Diadema setosum } \\
\text { Holothuria pardalis }\end{array}$ & $\begin{array}{c}1 \\
1 \\
1 \\
49(v) \\
3(v)\end{array}$ & \\
\hline
\end{tabular}




\begin{tabular}{llc} 
Station No. & Species & $\begin{array}{c}\text { Nos in } \\
\text { transect }\end{array}$ \\
\hline DA3/99/61 & Comanthus parvicirrus & 1 \\
E West Lewis I. & Culcita schmideliana & \\
Dive, Coll: P. Morrison & Protoreaster nodulosus & $1(\mathrm{v})$ \\
$4 / 9 / 99 ~ 5 \mathrm{~m}$ & Amphipholis squamata & 2 \\
& Ophiactis cf. brachyura & 1 \\
& Ophiactis savignyi & 1 \\
& Macrophiothrix callizona & 1 \\
& Macrophiothrix sp. juv. & 1 \\
& Ophiothrix ciliaris & 2 \\
& Peronella cf. macroproctes & 1 \\
& Stichopus horrens & $4(\mathrm{v})$ \\
& Neothyonidium magnum & $1(\mathrm{v})$
\end{tabular}

DA3/99/62

$S$ East Lewis I.

Intertidal

Coll: S. Morrison

$5 / 9 / 99$

\section{DA3/99/63}

SW East Lewis I.

Dive, Coll: P. Morrison $5 / 9 / 99 \quad 1.5 \mathrm{~m}$

\section{DA3/99/64}

S West Lewis I.

Dive, Coll: P. Morrison 6/9/99 5m

\section{DA3/99/65}

SW Enderby I.

Dive, Coll: P. Morrison $6 / 9 / 99 \quad 14 \mathrm{~m}$
Astropecten cf. sumbawanus

Ophiactis cf. fuscolineata

Salmacis sp. (d)

Peronella sp. (d)

Holothuria hilla

Holothuria pardalis

Holothuria (Lessonothuria) sp. juv.

Synaptula recta

Nos in

Nos outside

transect

Diadema setosum

Holothuria pardalis

$1(\mathrm{v})$

(v)

1

1

$1(\mathrm{v})$

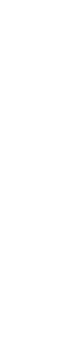


Table 4 (cont.)

\begin{tabular}{|c|c|c|c|}
\hline Station No. & Species & $\begin{array}{l}\text { Nos in } \\
\text { transect }\end{array}$ & $\begin{array}{l}\text { Nos outside } \\
\text { transect }\end{array}$ \\
\hline & $\begin{array}{l}\text { Peronella tuberculata (d) } \\
\text { Diadema setosum } \\
\text { Pseudocolochirus violaceus }\end{array}$ & $\begin{array}{c}1 \\
2(v)\end{array}$ & $1(v)$ \\
\hline $\begin{array}{l}\text { DA3/99/66 } \\
\text { S Enderby I., mangrove } \\
\text { Intertidal } \\
\text { coll: P. Morrison } \\
6 / 9 / 99\end{array}$ & $\begin{array}{l}\text { Ophionereis dubia } \\
\text { Peronella cf. macroproctes (d) } \\
\text { Actinopyga echinites } \\
\text { Holothuria atra } \\
\text { Holothria leucospilota }\end{array}$ & & $\begin{array}{l}2 \\
2 \\
\text { Few }(v) \\
\text { Few }(v) \\
\text { Few }(v)\end{array}$ \\
\hline $\begin{array}{l}\text { DA3/99/67 } \\
\text { N Nelson Rocks } \\
\text { Dive, Coll: P. Morrison } \\
7 / 9 / 99 \quad 17 \mathrm{~m}\end{array}$ & $\begin{array}{l}\text { Crinoid, black, hooks on pinnules } \\
\text { Comanthina variabilis } \\
\text { Linckia multifora } \\
\text { Acanthaster planci } \\
\text { Nardoa cf. galatheae } \\
\text { Culcita schmideliana (juv.) } \\
\text { Amphioplus stenaspis } \\
\text { Ophiactis modesta } \\
\text { Ophiactis savignyi } \\
\text { Ophiothrix ciliaris } \\
\text { Ophioconis cincta } \\
\text { Ophionereis intermedia } \\
\text { Phyllacanthus longispinus } \\
\text { Diadema setosum } \\
\text { Echinostrephus molaris } \\
\text { Holothuria edulis }\end{array}$ & $\begin{array}{l}1(\mathrm{v}) \\
1 \\
6(\mathrm{v}) \\
1(\mathrm{v}) \\
2 \\
1 \\
1 \\
1 \\
1 \\
1 \\
1 \\
3 \\
10(\mathrm{v}) \\
2(\mathrm{v})\end{array}$ & $\begin{array}{c}\text { Several(v) } \\
1(\mathrm{v}) \\
1 \\
\\
\operatorname{Few}(\mathrm{v})\end{array}$ \\
\hline $\begin{array}{l}\text { DA3/99/68 } \\
\text { SSW Nelson Rocks } \\
\text { Dive, Coll: P. Morrison } \\
7 / 9 / 99 \quad 6.5 \mathrm{~m}\end{array}$ & $\begin{array}{l}\text { Comanthina variabilis } \\
\text { Stephanometra spinipinna } \\
\text { Crinoid, black and white, swam } \\
\text { Crinoid, black, hooks on pinnules } \\
\text { Astropecten sp.? } \\
\text { Culcita schmideliana juv. } \\
\text { Macrophiothrix lineocaerulea } \\
\text { Ophiomaza cacaotica } \\
\text { Ophiothrix martensi } \\
\text { Ophiodaphne formata } \\
\text { Peronella lesueuri } \\
\text { Peronella sp. (d) } \\
\text { Nacospatangus interruptus (d) } \\
\text { Brissus latecarinatus } \\
\text { Rhynobrissus hemiasteroides } \\
\text { Cercodemas anceps } \\
\text { Synaptula recta }\end{array}$ & $\begin{array}{c}2 \\
1 \\
1(\mathrm{v}) \\
6(\mathrm{v}) \\
1(\mathrm{v}) \\
\\
1 \\
1 \\
1 \\
1 \\
7(\mathrm{v}) \\
1 \\
6 \\
1 \\
1 \\
5(\mathrm{v})\end{array}$ & $1(v)$ \\
\hline $\begin{array}{l}\text { DA3/99/69 } \\
\text { Pipe-line } \\
\text { Dive, Coll: P. Morrison } \\
8 / 9 / 99 \quad 14 \mathrm{~m}\end{array}$ & $\begin{array}{l}\text { Crinoid, green with yellow tips, white under } \\
\text { pinnules, hooks } \\
\text { Crinoid, as above, but brown } \\
\text { Crinoid, as above, but light green } \\
\text { Diadema setosum } \\
\text { Peronella cf. macroprotes }\end{array}$ & $\begin{array}{l}8(v) \\
3(v) \\
1(v) \\
13(v)\end{array}$ & 4 \\
\hline $\begin{array}{l}\text { DA3/99/70 } \\
\text { SW Nelson Rocks } \\
\text { Dive, Coll: M. Hewitt } \\
8 / 9 / 996 \mathrm{~m}\end{array}$ & $\begin{array}{l}\text { Crinoid, green, many arms, hooks } \\
\text { Crinoid, black } \\
\text { Crinoid, black with yellow tips } \\
\text { Crinoid, orange with yellow tips } \\
\text { Amphiutra sp. } \\
\text { Ophiothrix ciliaris } \\
\text { Ophiothrix exigua } \\
\text { Peronella cf. macroproctes (d) } \\
\text { Holothuria atra } \\
\text { Holothuria leucospilota } \\
\text { Leptopentacta grisea }\end{array}$ & $\begin{array}{l}2(\mathrm{v}) \\
4(\mathrm{v}) \\
1(\mathrm{v}) \\
1(\mathrm{v}) \\
1 \\
2 \\
1 \\
2 \\
2(\mathrm{v}) \\
1(\mathrm{v}) \\
1\end{array}$ & \\
\hline
\end{tabular}


Table 5 Echinoderms from the Dampier Archipelago collected by the dredging expedition (DA2/99); species, station numbers and depth range at which they were collected. $(v=$ visual or photographic record; $d=d e a d$ specimen).

\begin{tabular}{lllll}
\hline & Depth 5-10 $\mathrm{m}$ & Depth 11-20 m & Depth 21-30 m & Depth 31-40m \\
\hline ECHINODERMATA &
\end{tabular}

\section{Class CRINOIDEA}

\section{Family Comasteridae}

Clarkcomanthus littoralis (Carpenter, 1888)

Comanthina nobilis (Carpenter, 1884)

C. variabilis (Bell, 1882)

18

Comanthus alternans (Carpenter, 1881)

C. parvicirrus (Müller, 1841)

C. wahlbergi (Müller, 1843)

Comanthus sp. juv.

Comaster sp.

Comatula pectinata (Linnaeus, 1758)

C. purpurea (Müller, 1843)

C. rotalaria Lamarck, 1816

C. solaris Lamarck, 1816

Family Zygometridae

Zygometra elegans (Bell, 1882)

Z. microdiscus (Bell, 1882)

Z. punctata A.H. Clark, 1912

Family Himerometridae

Heterometra crenulata (Carpenter, 1882)

Himerometra robustipinna (Carpenter, 1881)

Family Colobometridae

Oligometra carpenteri (Bell, 1884)

Oligometrides adeonae (Lamarck, 1816)

Family Antedonidae

Toxometrasp.

Crinoidea spp indet.

\section{Class ASTEROIDEA}

\section{Family Luidiidae}

Luidia hardwicki (Gray, 1840)

L. maculata Müller and Troschel, 1842

Family Astropectinidae

\section{Astropecten granulatus Müller and}

Troschel, 1842

A. monacanthus Sladen, 1883

A. polyacanthus Müller and Troschel, 1842

A. vappa Müller and Troschel, 1843

A. velitaris von Martens, 1865

91

A. zebra Sladen, 1883

Family Archasteridae

Archaster angulatus Müller and Troschel, 1842

70
71

$1,70,71,72$

$1,67,68,91$

33

$$
41
$$

$33,34,59$

$21,40,49$

$41,46,49,60,83$

$21,34,38,39,41$, $46,69,73,83$

$46,49,74,76$

$34,35,36,38,39$, $0,59,60,75$
9,57

58

9

50,85

6,9

6

7,9

$4,6,9$

$6,12,23$

24

5,9

$6,12,23,24$

$8,50,58$
$8,10,50,58$
32,99

13,99

50

44

6,7

13

$32 \mathrm{v}, 38 \mathrm{v}, 49 \mathrm{v}$

$30 \mathrm{v}, 43 \mathrm{v}, 47 \mathrm{v}, 48 \mathrm{v} \quad 25 \mathrm{v}, 26 \mathrm{v}, 52 \mathrm{v}$, $54 \mathrm{v}, 55,56 \mathrm{v}$

$65,75,83,84$

$65,75,83,84$
$21,32 \mathrm{v}, 40 \mathrm{v}, 42 \mathrm{v}$

30,47

$25,26,55$

$58 \mathrm{v}$

$5,7,24 \mathrm{v}, 26$,

$51 \mathrm{v}, 52 \mathrm{v}, 54$,

$55,56 \mathrm{v}$

28

12,22

54,56

$8 \mathrm{v}, 28,29,30,44, \quad 4,12,22,23$,

47,48

28,29

$26,54,56$

$3,4,54,55$

58

78

$18 \mathrm{v}, 19 \mathrm{v}, 71,72 \quad 32,33,38,39$,

$40,41,74$

32

44

$6,22,26,27,55$

44

12,87 
Table 5 (cont.)

\begin{tabular}{|c|c|c|c|c|}
\hline & Depth 5-10 m & Depth 11-20 m & Depth 21-30 m & Depth $31-40 \mathrm{~m}$ \\
\hline S. princeps Sladen, 1889 & & $21 v, 33,40,69$ & $8 v$ & $\begin{array}{l}4,7,9,12 \\
57,81\end{array}$ \\
\hline S. squamulosus (Studer, 1884) & & & 58 & 55,57 \\
\hline
\end{tabular}

Family Oreasteridae

Anthenea conjungens Döderlein, 1935

A. elegans H.L. Clark, 1938

Anthenea sp. juv.

Goniodiscaster acanthodes H.L. Clark, 1938 72,91

G. australiae Tortonese, 1937

Gymnanthenea globigera (Döderlein, 1915)

Pentaceraster gracilis (Lütken, 1871)

$P$. sp. cf. regulus Müller and Troschel, 1842 juv.

Pentaceraster sp. juv.

Pseudoreaster obtusangulus (Lamarck, 1816)

75,99

41

$33,73,76$

84

10

45

Family Ophidiasteridae

Hacelia helicosticha (Sladen, 1889)

Tamaria hirsuta (Koehler, 1910)

T. tumescens (Koehler, 1910)

\section{Family Pterasteridae}

Euretaster insignis (Sladen, 1882)

Family Asterinidae

Anseropoda rosacea (Lamarck, 1816)

62,70

73

Nepanthia belcheri (Perrier, 1875)

N. maculata Gray, 1840

\section{Family Echinasteridae}

Echinaster varicolor H.L. Clark, 1938

Metrodira subulata Gray, 1840

70

$18,71,72$
34,73

75

$32,33,41,73,74,75$
$4,5,6,55,57$

$5,7,9$

88

54,55

69

4,7
$54,57,81$ $4,54,55,56$ 79,81

8

\section{9}

54

Gen. and sp. indet.

Family Ophiactidae

Ophiactis brevis H.L. Clark, 1938

O. luteomaculata H.L. Clark, 1915 
Depth 5-10 m

O. modesta Brock, 1888

O. savignyi (Müller and Troschel, 1842)

$18,68,70$

Ophiactis sp.

Ophiodaphne formata Koehler, 1905

O. scripta (Koehler, 1904)

\section{Family Ophiotrichidae}

Macrophiothrix 'caenosa' sensu

Hoggett, 1990

M. lineocaerulea (H.L. Clark, 1928)

M. megapoma H.L. Clark, 1938

M. melanosticta (Grube, 1868)

M. microplax Bell, 1884

$M$. variabilis (Duncan, 1887)

Macrophiothrix sp. cf. variabilis (Duncan, 1887) juv.

Macrophiothrix sp. juv.

Ophiocnemis marmorata (Lamarck, 1816)

Ophiomaza cacaotica Lyman, 1871

Ophiothela danae Verrill, 1869

Ophiothrix (Ophiothrix) ciliaris (Lamarck, 1816)

O. (O.) sp. cf. comata Müller and Troschel, 1842

O. (O.) exigua Lyman, 1874

O. (O.) plana Lyman, 1874

$O$. (O.) plana group

Ophiothrix (O.) sp. juv.

O. (Acanthophiothrix) sp. cf. armata

Koehler, 1905

O. (A.) sp. juv.

O. (A.) viridialba von Martens, 1867

O. (Keystonea) martensi Lyman, 1874

O. (K.) smaragdina Studer, 1882

Family Ophionereididae

Ophionereis dubia (Müller and

Troschel, 1842)

Family Ophiodermatidae

Ophiarachnella gorgonia (Müller and

Troschel, 1842)

O. megaloplax Bell, 1884

O. similis (Koehler, 1905)

Ophiarachnella sp. ? nov.

Ophiochasma stellata (Ljungman, 1867)

Ophioconis cincta Brock, 1888

Ophiopsammus yoldii (Lütken, 1856)

Ophiopsammus sp. cf. yoldii (Lütken, 1856

Family Ophiuridae

Dictenophiura stellata (Studer, 1882)

Ophiolepis unicolor H.L. Clark, 1938

Ophitura kinbergi (Ljungman, 1866)

\section{Class ECHINOIDEA}

\section{Family Cidaridae}

Phyllacanthus longispinus Mortensen, 1918

18

$18,68,91$

$68,70,91,95$

62

70

68,91

67

$18,62,68,70,72$,

91,95
68

Depth 11-20 m

Depth 21-30 m

Depth 31-40m

75

$37,42,65,76,77$,

$8,10,50,58$

$6,9,22$

84,99

13

46

47

$13,33,37,42,77,99$

$8,50,58$

82,90

$37,73,75,99$

$10,58,85$

79,90

$13,37,60,73,75$

$8,10,50,58$

6

$76,77,84,99$

$21,46,49,78$

$43,44,48$

$6,22,23,25$,

$76,77,84$

42

73,42

$42,49,73$

21,41

77

$26,27,54,56$

$6,9,82$

$13,31,33,37,39$,

$42,59,60,65,73,58,85$

$75,77,84,94,98,99$

31

$18,70,95$

18

18

$37,39,65,73,84$

$37,39,65,84$

99

$49,69,75$

39,69

46

91,95

62,95

$13,31,59,65,75$,
$31,73,74,77,84,99 \quad 43,58,85$

$77,84,98,99$

73

13

$18,62,70$

$21,49,73,78$

$33,69,74$

85

13,77

$33,34,73,75$

69

45

8
$4,23,79,82$
$6,24,26$
7

$4,6,9,55$

$\begin{array}{ll}30,43,47 & 4,23,79,82 \\ 58 & 6,24,26 \\ & 7 \\ 8,10,29,48, & 4,6,9,55\end{array}$

$6,22,90$

$8,10,50,58,85 \quad 88$
4

82

4

5,9

9

$6,22,90$
$41,42,69$
99

41
$6,9,12,23,24$

88

7

$4,6,7,22,23$,

$24,25,26,52$,

$54,55,56,57$,

81,90

$6,25,26$

7

4 
Table 5 (cont.)

\begin{tabular}{lllll}
\hline & Depth 5-10 m & Depth 11-20 m & Depth 21-30 m & Depth 31-40m \\
\hline Prionocidaris baculosa (Lamarck, 1816) & $96 \mathrm{~d}$ & 33d, 35d & $29 \mathrm{~d}, 58$ & $3 \mathrm{~d}, 4 \mathrm{~d}, 5 \mathrm{~d}, 7 \mathrm{~d}$, \\
& & & & $12 \mathrm{~d}, 88 \mathrm{~d}$ \\
P. bispinosa (Lamarck, 1816) & $1,91,96 \mathrm{~d}$ & $21,32,35 \mathrm{~d}, 69$, & 10 & $3 \mathrm{~d}, 4,5 \mathrm{~d}, 7,9$, \\
& & 73,75 & & $22,57,81 \mathrm{~d}, 87 \mathrm{~d}$
\end{tabular}

Family Diadematidae

Chaetodiadema granulatum Mortensen, 1903

55

Family Temnopleuridae

Salmacis belli Döderlein, 1902

S. sphaeroides (Linnaeus, 1758)

Temnopleurus alexandri (Bell, 1884)

Temnopleurus decipiens (de Meijere, 1904)

Temnotrema siamense Mortensen, 1904

$$
62,71
$$

21,75

35

6,52

62,91

62

40,60

Family Toxopneustidae

Nudechinus darnleyensis (Tenison-Woods, 1878)

$62,91 \quad 21,73,76$

Family Clypeasteridae

Clypeaster latissimus (Lamarck, 1816)

C. telurus H.L. Clark, 1914

71,72

Family Laganidae

Peronella lesueutri (Valenciennes, 1841)

$1,18,71,72 \mathrm{~d}, 95$

P. macroproctes Koehler, 1922

P. tuberculata Mortensen, 1918

Peronella sp. cf. orbicularis (Leske, 1778)

Peronella sp. (incomplete)

Peronella sp.

72

$1,18,72,91,95$

$19 \mathrm{~d}$

$68 \mathrm{~d}, 70 \mathrm{~d}, 71 \mathrm{~d}, 96 \mathrm{~d}$

$32,41,49,76$

48

58

$4,5,12,56$

$6,9,23$

$32,35,37,39,40$,

$41,49,60 \mathrm{~d}, 69,75$

$73,76,78$

$40,41,49,69$

$2 \mathrm{~d}$

29,47

$4 d, 22 v, 53 d$

29

29,47

$47 \mathrm{~d}$

$47 d$

$49,83 \mathrm{~d}$

49

18,71

$41,46,49$

48

$46,78 \mathrm{~d}$

$61 d$

Schizaster (Schizaster) compactus

(Koehler, 1914)

\section{Family Brissidae}

Brissopsis sp. aff. persica Mortensen, 1940

Metalia sternalis (Lamarck, 1816)

cf. Rhynobrissus sp.

Family Loveniidae

Breynia desorii Gray, 1851

Lovenia elongata (Gray, 1845)

Family Spatangidae

Nacospatangus (Pseudomaretia) interruptus (Studer, 1880)

\section{Class HOLOTHUROIDEA}

\section{Family Holothuriidae}

Actinopyga echinites (Jaeger, 1833)

Holothuria Halodeima) edulis Lesson, 1830

Holothuria (Metriatyla) sp. aff. albiventer

Semper, 1868

Holothurria (Metriatyla) scabra Jaeger, 1833
77

75

$33 \mathrm{~d}, 36 \mathrm{~d}, 37,39,59$

$60 \mathrm{~d}, 69,75,76,94$

$31,46,78,83$

47

$4,7,24,51$,

52,53

$33 d, 46,49,76$

58,89

57
$55 d, 57$

\section{$12,26 \mathrm{~d}, 27 \mathrm{~d}$}

$4 d, 7 d, 9 d, 22 d$

7

12 


H. (Metriatyla) timana Lesson, 1830
H. (Stauropora) sp. cf. modesta
Ludwig, 1875
H. (Theelothuria) michaelseni Erwe, 1913
H. (Theelothuria) spinifera Théel, 1886
H. (Thymiosycia) sp.

Family Stichopodidae

Stichopus chloronotus Brandt, 1835

S. horrens Selenka, 1867

Stichopus sp. juv.

\section{Family Cucumariidae}

Actinocucumis typica Ludwig, 1875

Cercodemas anceps Selenka, 1867

Colochirus crassus Ekman, 1918

C. quadrangularis Troschel, 1846

Leptopentacta grisea H.L. Clark, 1938

Leptopentactasp.

Loisettea amphictena Rowe and

Pawson, 1985

L. gazellae (Lampert, 1889)

Mensamaria intercedens (Lampert, 1885)

Plesiocolochirus australis (Ludwig, 1875)

$P$. challengeri (Théel, 1886)

Pseudocolochirus violaceus (Théel, 1886)

\section{Family Phyllophoriidae}

Havelockia versicolor (Semper, 1868)

Hemithyone semperi (Bell, 1884)

Phyllophorus (Phyllophorella) spiculata Chang, 1935

P. (Urodemella) brocki Ludwig, 1888

P. (Urodemella) proteus Bell, 1884

Phyrella sp. cf. trapeza (H.L. Clark, 1932)

Stolus buccalis (Stimpson, 1855)

S. canescens (Semper, 1868)

S. conjungens (Semper, 1868)

Thyone dura Koehler and Vaney, 1908

T. grisea H.L. Clark, 1938

Thyone papuensis Théel, 1886

Thyonesp. cf. papuensis Théel, 1886

Thyonesp. aff. theeli Rowe, 1995

Thyonesp.

Gen. and sp. indet. (1)

Gen. and sp. indet. (2)

Family Sclerodactylidae

Cladolabes schmeltzï (Ludwig, 1875)

\section{Family Caudinidae}

Acaudina leucoprocta (H.L. Clark, 1938)

A. sp. cf. molpadioides (Semper, 1868)

\section{Family Molpadiidae}

Molpadia scabrum (Sluiter, 1901)

Family Synaptidae

Synaptula recta (Semper, 1868)

Synaptula sp.

Gen. and sp. indet. (incomplete specimen)

Depth 5-10 m

Depth $11-20 \mathrm{~m}$

Depth 21-30 m

Depth 31-40m

70

74

70,96

21,36
75

5,27
57

75

49

6

49

72

$1,18,67,70$

18,70

96

70

72

2

21
$39 v, 49 v$

31,59

$21,33,39,59,75$

21,75

$37,39,59,65,69,75$

50

$8,10,50$

$8,10,58 \mathrm{v}$

$21,33,75$

59

$65,75,94$

59

$8,10,50$

10

$44,45 \mathrm{v}, 47 \mathrm{v}$

10,44

$8,10,50$

44

50

7

$4,12,25 \mathrm{v}$

55,57

6,9

57

57

$4,6,24$

$56 \mathrm{v}$

4

6,9

$6,12,22,79$

4

6

59,94

$33,37,65$

69

$39,42,65,69,75,98$

37

39

8

$4,6,9,57,79$

57

55

10

55

26

4,12

21

10

41

34,35

28

$23,55,90$

$62,68,72$

$37,39,59,65,75$

8,10

6,9
88

24
68

91 
Table 6 Diving expedition (DA1/98, DA3/99) sites with the highest number of echinoderm species.

\begin{tabular}{lccl}
\hline Depth & $\begin{array}{c}\text { Site } \\
\text { no. }\end{array}$ & $\begin{array}{c}\text { Nos } \\
\text { species }\end{array}$ & \multicolumn{1}{c}{ Habitat } \\
\hline Intertidal & 45 & 24 & Loose igneous rocks on sand, brown algae, seagrass in pools. \\
Intertidal & 10 & 21 & Passage between islands, corals and algae. \\
Intertidal & 23 & 19 & Silty flat with live and dead coral. \\
$0-10 \mathrm{~m}$ & 22 & 23 & Hard corals, coral rubble and sand. \\
$0-10 \mathrm{~m}$ & 47 & 22 & Sand covered pavement with brown and green algae, a few hard corals. \\
$0-10 \mathrm{~m}$ & 16 & 21 & Coral rubble, algae, some live coral. \\
$11-30 \mathrm{~m}$ & 65 & 31 & Sand covered pavement with sponges, gorgonians, hard and soft corals. \\
$11-30 \mathrm{~m}$ & 52 & 18 & Steep rock slope with hard and soft corals and gorgonians. \\
$11-30 \mathrm{~m}$ & 55 & 18 & Silty sand with sponges, soft corals and gorgonians. Strong current. \\
\hline
\end{tabular}

Table 7 Dredging expedition (DA2/99) sites with the highest number of echinoderm species.

\begin{tabular}{lccl}
\hline Depth & $\begin{array}{c}\text { Site } \\
\text { no. }\end{array}$ & $\begin{array}{c}\text { Nos } \\
\text { species }\end{array}$ & \multicolumn{1}{c}{ Habitat } \\
\hline $5-10 \mathrm{~m}$ & 70 & 20 & Gravel and rocks with gorgonians, tunicates, corals. \\
$5-10 \mathrm{~m}$ & 18 & 19 & Grey muddy sand with hydroids and gorgonians. \\
$5-10 \mathrm{~m}$ & 91 & 18 & Sandy mud with shell rubble, algae, gorgonians and tunicates. \\
$11-20 \mathrm{~m}$ & 75 & 29 & Muddy sand and rock, brown algae, sponges, gorgonians and soft corals. \\
$11-20 \mathrm{~m}$ & 73 & 23 & Coarse sand and rocks, sponges, free-living corals. \\
$11-20 \mathrm{~m}$ & 49 & 22 & Rock, coarse sand, green and brown algae, sponges, tunicates. \\
$21-30 \mathrm{~m}$ & 8 & 21 & Fine sand, shelly gravel, limestone rocks, sponges, gorgonians, soft corals and tunicates. \\
$21-30 \mathrm{~m}$ & 10 & 19 & Coarse shelly sand and limestone rocks, sponges, gorgonians and soft corals. \\
$21-30 \mathrm{~m}$ & 58 & 19 & Coarse shelly gravel, rocks, red and brown algae, hydroids. \\
$31->40 \mathrm{~m}$ & 6 & 29 & Coarse muddy sand, limestone rocks, sponges, gorgonians. \\
$31->40 \mathrm{~m}$ & 4 & 25 & Muddy shelly sand, rubble, limestone rocks, sponges and gorgonians. \\
$31->40 \mathrm{~m}$ & 9 & 24 & Coarse sand, limestone rocks, sponges, gorgonians, soft corals and tunicates. \\
\hline
\end{tabular}


Table 8 Total number of echinoderm species from each station of the diving expeditions, DA1/98 (stns 1-35) and DA 3/99 (stns 36-70), grouped by depth range.

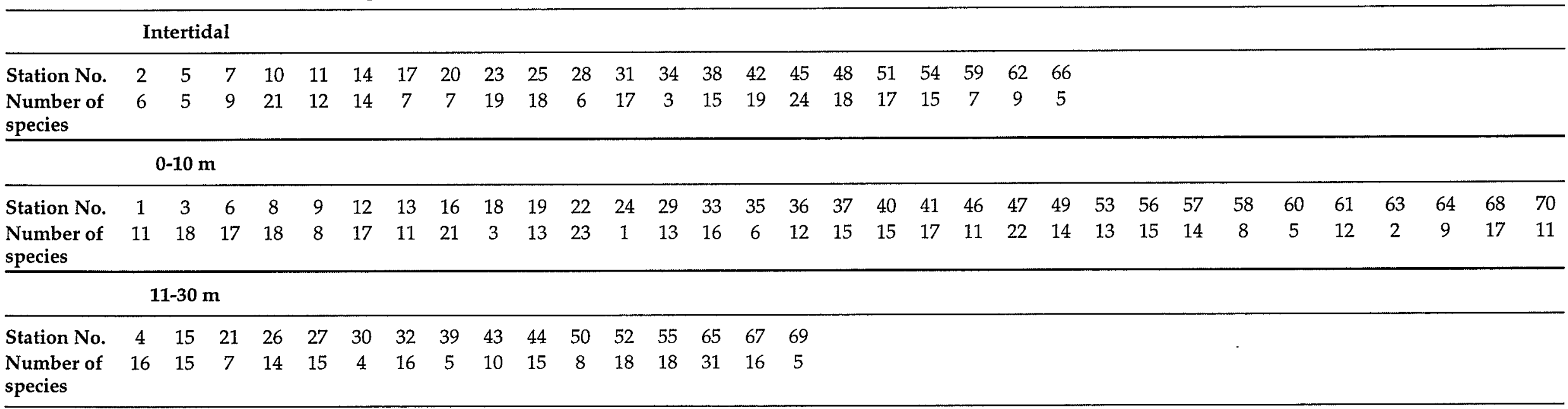

Table 9 Total number of echinoderm species from each station of the dredging expedition (DA2/99) grouped by depth range.

$$
\text { 5-10 m }
$$

\begin{tabular}{lllllllllllllll}
\hline Station No. & 1 & 15 & 18 & 19 & 62 & 66 & 67 & 68 & 70 & 71 & 72 & 91 & 95 & 96
\end{tabular}

$\begin{array}{lllllllllllllll}\text { Number of } & 9 & 4 & 19 & 3 & 12 & 1 & 4 & 13 & 20 & 12 & 16 & 18 & 7 & 10\end{array}$

species

\section{$11-20 \mathrm{~m}$}

$\begin{array}{llllllllllllllllllllllllllllllllllllllllllllll}\text { Station No. } & 2 & 13 & 16 & 17 & 20 & 21 & 31 & 32 & 33 & 34 & 35 & 36 & 37 & 38 & 39 & 40 & 41 & 42 & 46 & 49 & 59 & 60 & 61 & 65 & 69 & 73 & 74 & 75 & 76 & 77 & 78 & 83 & 84 & 94 & 98 & 99\end{array}$ $\begin{array}{lllllllllllllllllllllllllllllllllllllllll}\text { Number of } & 1 & 19 & 2 & 2 & 1 & 19 & 7 & 10 & 18 & 8 & 6 & 4 & 15 & 4 & 17 & 10 & 19 & 11 & 13 & 22 & 13 & 9 & 2 & 11 & 18 & 23 & 7 & 29 & 11 & 11 & 6 & 6 & 10 & 5 & 3 & 15\end{array}$ species

21-30 m

\begin{tabular}{lllllllllllllllll}
\hline Station No. & 8 & 10 & 18 & 28 & 29 & 30 & 43 & 44 & 45 & 47 & 48 & 50 & 58 & 85 & 89
\end{tabular}

$\begin{array}{llllllllllllllll}\text { Number of } & 21 & 19 & 1 & 4 & 7 & 5 & 5 & 8 & 4 & 12 & 6 & 14 & 19 & 6 & 1\end{array}$

species

\begin{tabular}{|c|c|c|c|c|c|c|c|c|c|c|c|c|c|c|c|c|c|c|c|c|c|c|c|c|c|c|}
\hline & & -43 & & & & & & & & & & & & & & & & & & & & & & & & \\
\hline Station No. & 3 & 4 & 5 & 6 & 7 & 9 & 12 & 22 & 23 & 24 & 25 & 26 & 27 & 51 & 52 & 53 & 54 & 55 & 56 & 57 & 79 & 81 & 82 & 87 & 88 & 90 \\
\hline $\begin{array}{l}\text { Number of } \\
\text { species }\end{array}$ & 3 & 25 & 11 & 29 & 17 & 24 & 17 & 11 & 10 & 9 & 6 & 11 & 4 & 3 & 5 & 2 & 11 & 17 & 10 & 19 & 5 & 5 & 5 & 2 & 6 & 5 \\
\hline
\end{tabular}

species 


\section{DA $1 / 98$}

DA 2/99

DA $3 / 99$

\section{ECHINODERMATA}

\section{Class CRINOIDEA}

\section{Family Comasteridae}

Clarkcomanthus littoralis (Carpenter, 1888)

Comanthina nobilis (Carpenter, 1884)

C. gisleni Rowe et al., 1986
C. variabilis (Bell, 1882)

Comanthus alternans (Carpenter, 1881)

C. briareus (Bell, 1882)

C. parvicirrus (Müller, 1841)

C. wahlbergi (Müller, 1843)

Comanthus sp.

Comaster sp.

Comatella maculata (Carpenter, 1888)

C. stelligera (Carpenter, 1888)

Comatula pectinata (Linnaeus, 1758)

C. purpurea (Müller, 1843)

C. rotalaria Lamarck, 1816

C. solaris Lamarck, 1816

Oxycomanthus sp.

$10,25,33$

\section{Family Zygometridae}

Zygometra comata A.H. Clark, 1911

Z. microdiscus (Bell, 1882)

Z. punctata A.H. Clark, 1912

\section{Family Himerometridae}

Amphimetra tessellata (Müller, 1841)

Heterometra crenulata (Carpenter, 1882)

Himerometra robustipinna (Carpenter, 1881)

\section{Family Mariametridae}

Lamprometra palmata (Müller, 1841)

S. spinipinna (Hartlaub, 1890)

\section{Family Colobometridae}

Colobometra perspinosa (Carpenter, 1881)

cf. Iconometra sp.

Oligometra carpenteri (Bell, 1884)

O. serripinna (Carpenter, 1881)

$16,22,25$.
Z. elegans (Bell, 1882)

Stephanometra indica (Smith, 1876)

S. oxyacantha (Hartlaub, 1890)
$4,13,21$
9,57

18

$9,13,33,41,58,62,99$

$6,9,13,49,50,73,85$

15

$7,8,13,15,41,42,50,58,69,73,99$

$4,6,8,9,10,13,15,42,50,58,70$

$6,12,21,23$

$18,21,24,41,46,68,73$
$36,47,48,52$

$37,41,67,68$

55

$37,49,65$

36,61

45,65

36,47

57

55,65

49,65

55

$5,9,18,60,69$

$6,12,23,24,32,99$

$13,50,99$

55

55

31,44

99

47

$40,46,50$

40,68

55

$52,55,65$ 


\section{Family Antedonidae}

Dorometra parvicirra (Carpenter, 1888)

\section{Class ASTEROIDEA}

Family Luidiidae

Luidia hardwicki (Gray, 1840)

L. maculata Müller and Troschel, 1842

$77,25,26,30,36,39,40,47,60,65,75,83,84$ $5,7,21,26,54,55$

\section{Family Astropectinidae}

Astropecten granulatus (Müller and

A. monacanthus Sladen, 1883

A. polyacanthus Müller and Troschel, 1842

A. sumbawanus Döderlein, 1917

$12,22,28,4$

A. vappa Müller and Troschel, 1843

$21,40,49,54,56,91$

$4,12,22,23,26,28,29,30,41,44,46,47,48,49,54$ $56,60,71,83$

$1,3,4,28,29,34,38,39,41,46,54,55,69,70,71$

$$
72,73,83
$$

Family Archasteridae

Archaster angulatus Müller and Troschel, 1842

$49,58,70,74,76$

\section{Family Goniasteridae}

Iconaster longimanus (Möbius, 1859)

Stellaster equestris (Retzius, 1805)

S. inspinosus H.L. Clark, 1916

S. princeps Sladen, 1889

S. squamulosus (Studer, 1884)

Styphlaster notabilis H.L. Clark, 1938

78

$6,22,26,27,32,33,38,40,41,44,55,71,72,74$

$12,32,44,81$

$4,7,9,12,33,40,57,69,81$

$55,57,58$

\section{Family Oreasteridae}

Anthenea conjungens Döderlein, 1935

A. elegans H.L. Clark, 1938

Anthenea sp. juv.

Culcita schmideliana (Retzius, 1805)

\section{5,99}

41

$3,4,10,14,16,17,25$

10

Goniodiscaster acanthodes H.L. Clark, 1938

G. australiae Tortonese, 1937

Gymnanthenea globigera (Döderlein, 1915)

Pentaceraster gracilis (Lütken, 1871) $27,32,33$

$33,57,72,73,76,91$
$5,10,12,33,82$
45,84
$5,6,12,18,23$
58
57

$36,37,43,45,48,52,56,57,58,61$, $65,67,68$

65

45,48 
Protoreaster lincki (Blainville, 1830)

$P$. nodulosus (Perrier, 1875)

Pseudoreaster obtusangulus (Lamarck, 1816)

Family Ophidiasteridae

Fromia indica (Perrier, 1869)

Fromia sp. ? nov.

Gomophia sphenisci (A.M. Clark, 1967)

Hacelia helicosticha (Sladen, 1889)

Linckia laevigata (Linnaeus, 1758)

L. multifora (Lamarck, 1816)

Nardoa galatheae (Lütken, 1865)

Ophidiaster sp.

Tamaria hirsuta (Koehler, 1910)

T. tumescens (Koehler, 1910)

Tamaria sp.

Family Pterasteridae

Euretaster insignis (Sladen, 1882)

Family Asterinidae

Anseropoda rosacea (Lamarck, 1816)

Asterina anomala H.L. Clark, 1921

A. sarasini (de Loriol, 1879)

Asterina sp. ? nov.

Nepanthia belcheri (Perrièr, 1875)

N. maculata Gray, 1840

Family Acanthasteridae

Acanthaster planci (Linnaeus, 1758)

Family Echinasteridae

Echinaster superbus H.L. Clark, 1916

E. varicolor H.L. Clark, 1938

Metrodira subulata (Gray, 1840)

\section{Class OPHIUROIDEA}

Family Euryalidae

Euryale asperum Lamarck, 1816

\section{Family Amphiuridae}

Amphioplus (Amphichilus) ochroleuca

(Brock, 1888)

\section{1,26}

15,26

$3,4,15,21,26,27$

75,82

72,73

$69,172,73,75$

$62,70,73$

31

$4,16,18,26,32$

17

$62,70,75$

$4,5,6,18,32,33,41,55,57,71,72,73,74,75$

$9,37,39,59,75$

34,73
32,74

47

51,61

$41,45,48,51,54$

52,57

65

57

52,57

43,56

39

$43,47,67$

no loc.

55

no loc.

64

$36,40,43,44,46,49,50,52,67$

no loc.

56,57 
A. (Amphioplus) lucidus Koehler, 1922

A. (Amphioplus) stenaspis H.C. Clark, 1938

A. (Lymanella) depressa (Ljungman, 1867)

A. (Lymanella) sp.

Amphipholis squamata (Delle-Chiaje, 1828)

Amphiura (Amphiura) acrisia H.L. Clark, 1938

A. (Amphiura) bidentata H.L. Clark, 1938

A. (Amphiura) catephes H.L. Clark, 1938

A. (Amphiura) constricta Lyman, 1879

A. (Amphiura) diacritica H.L. Clark, 1938

A. (Amphiura) duncani Lyman, 1882

A. (Amphiura) morosa Koehler, 1905

A. (Amphiura) sp. cf. morosa Koehler, 1905

A. (Amphiura) phrixocantha Clark and

Clark, 1976

A. (Amphiura) velox Koehler, 1910

A. (Amphiura) sp. aff. velox Koehler, 1910

A. (Amphiura) sp.

Dougaloplus echinatus (Ljungman, 1867)

Ophiocentrus dilatata (Koehler, 1905)

O. sp. cf. dilatata (Koehler, 1905)

Family Ophiactidae

Ophiactis sp. cf. brachyura Döderlein, 1898

O. brevis H.L. Clark, 1938

O. fuscolineata H.L. Clark, 1938

Ophiactis sp. cf. fuscolineata H.L. Clark, 1938

O. luteomaculata H.L. Clark, 1915

O. macrolepidota Marktanner-

Turneretscher, 1887

O. modesta Brock, 1888

Ophiactis sp. cf. modesta Brock, 1888

O. savignyi (Müller and Troschel, 1842)

Ophiactis sp. cf. savignyi (Müller and

Troshel, 1842)

O. versicolor H.L. Clark, 1939

Ophiactis sp.

Ophiodaphne formata (Koehler, 1905)

O. scripta (Koehler, 1904)

$1,3,4,13,33$

8,11

4

29,33

33

\section{$2,7,17,23$}

$6,8,9,10,12,13,16,29,32$

$10,28,31,33$

$13,27,32$

$1,3,4,5,6,8,10,12,13,15,6,8,9,10,18,22,37,42,50,58,65,68,70$ $16,17,20,22,23,25,26,27, \quad 76,77,84,99$

$29,31,32,33,34,35$

22,31

1,22
13

$4,54,55,56,79,81$

10,76
21
96
8,9

47

$9,18,57$

75
$53,59,61$

$40,41,53,65,66$
47,62

$52,55,65,66,67$

$37,38,45,46,48,49,53,56,60,61,67$

$37,40,58$

41,68 


\section{Family Ophiotrichidae}

Macrophiothrix caenosa sensu Hoggett, 1990

M. callizona H.L. Clark, 1938

M. lineocaerulea (H.L. Clark, 1928)

M. lorioli A.M. Clark, 1968

M. megapoma H.L. Clark, 1938

$8,13,33,37,42,50,58,77,82,90,98,99$

$10,18,37,58,68,73,75,79,85,80,91,99$

$6,10,13,37,50,58,60,68,70,73,75,76,77$

$84,91,95,99$

$6,21,22,23,25,26,27,43,44,46,48,49,54,56,62,78$ 70

M. melanosticta (Grube,

M. paucispina Hoggett, 1991

$M$. variabilis (Duncan, 1887)

Macrophiothrix sp. juv.

Ophiocnemis marmorata (Lamarck, 1816)

Ophiomaza cacaotica Lyman, 1871

Ophiopteron elegans Ludwig, 1888

Ophiothela danae Verrill, 1869

Ophiothrix (Ophiothrix) ciliaris (Lamarck, (1816)

O. (Ophiothrix) sp. cf. comata Müller and Troschel, 1842

O. (Ophiothrix) exigua Lyman, 1874

O. (Ophiothrix) plana Lyman, 1874

Ophiothrix (Ophiothrix) sp. juv.

$O$. (Acanthophiothrix) sp. cf. armata

Koehler, 1905

O. (Acanthophiothrix) viridialba von

Martens, 1867

Ophiothrix (Keystonea) martensi Lyman, 1874

$O$. (Keystonea) smaragdina Studer, 1882

$10,12,15,23,27,31$

10,15

$9,10,11,14,19$

$3,6,8,32$

30

$8,13,15,27,31$

1,3,4,6,7,8,9,10,12,13,

$15,17,19,21,22,25,26,27$

$29,31,32,33$

$3,5,7,10,12,13,19,25$, 27,31

$5,6,7,8,10,11,12,13$,

$15,16,17,19,20,22,23$,

$25,26,27,29,31,33$

1,10

Family Ophiocomidae

Ophiocoma dentata Müller and Troschel, $1842 \quad 14,16$

O. sp. cf. pusilla (Brock, 1888)

Ophiomastix mixta Lütken, $1869 \quad 1,22$

O. variabilis Koehler, 1905

\section{Family Ophionereidida}

Ophionereis dubia (Müller and Troschel, 1842) 10, 14
$6,9,68,76,77,82,84$

42,73

$4,23,30,42,43,47,49,73,79,82$

$6,21,26,41,58$

$7,67,77$

源

$58,59,60,62,65,68,70,72,73,75,84,85,91,98,99$

31

$4,8,18,37,39,65,70,73,84,95$

$8,37,39,65,82,84$

69

$43,45,46,47$

$6,22,31,43,58,73,74,77,84,85,90,91,95,99$

$8,10,13,31,50,58,59,62,65,75,77,84,88,95,98,99$
$37,38,40,42,45,47,49,64,65$

$41,42,61$

$38,42,54$

48,61

$41,43,65$

$36,37,38,40,41,47,48,51,53,55,56,58$,

$60,61,65,67,70$

$40,41,42,55,56,57,60,65,70$

$36,40,43,46,51,52,53,54,64$

$41,46,49,53,65$

$39,55,56,57,65$ 


\section{Family Ophiodermatidae}

Ophiarachnella gorgonia (Müller and

Troschel, 1842)

O. infernalis (Müller and Troschel, 1842)

$8,20,21$

aloplax Bell, 1884

O. similis (Koehler, 1905)

O. sphenisci (Bell, 1894)

Ophiarachnella sp. 1

Ophiochasma stellata (Ljungman, 1867)

Ophioconis cincta Brock, 1888

O. cupida Koehler, 1905

Ophiodyscrita acosmeta H.L. Clark, 1938

Ophiopsammus yoldii (Litken, 1856)

Ophiopsammus sp. cf. yoldii (Lütken, 1856)

16,20

$6,9,12,18,21,23,24,30,49,62,70,73,78$

$42,49,54$

$4,6,7,22,23,25,26,33,52,54,55,56,57,69,74,85,90$

$$
13,77
$$

$8,12,33$

$6,7,8,25,26,33,34,45,70,73,75$

69

22

Dictenophiura stellata (Studer, 1882)

Ophiolepis unicolor H.L. Clark, 1938

Ophioplocus imbricatus (Müller and

Troschel, 1842)

Ophiura kinbergi (Ljungman, 1866)

$4,6,8,14,16$

\section{$42,68,69$}

4,99

$42,47,48,53,65$

$37,45,46,47,48,51,53,65$

41

\section{Class ECHINOIDEA}

Family Cidaridae

Phyllacanthus imperialis (Lamarck, 1816)

P. longispinus Mortensen, 1918

Prionocidaris baculosa (Lamarck, 1816)

P. bispinosa (Lamarck, 1816)

$3,4,5,7,12,29,33,35,58,88,96$

$1,3,4,5,7,9,10,21,22,32,35,57,69,73$,

$75,81,87,91,96$

Family Diadematidae

Chaetodiadema granulatum Mortensen, 1903

Diadema setosum (Leske, 1778)

55

$1,3,4,6,8,12,15,16,18$ $19,21,22,26,27,29,32$

Family Temnopleuridae

Salmacis belli Döderlein, 1902

S. sphaeroides (Linnaeus, 1758)

Salmacis sp.

Temnopleurus alexandri (Bell, 1884)

$T$. decipiens (de Meijere, 1904)

Temnopleurus sp.

Temnotrema siamense Mortensen, 1904

23,25

9
6,52

$21,62,71,75$

35,56

60,91

40,62
$36,39,40,46,50,67$
$36,44,48,49,50,52,53,57,58$

$60,63,64,65,67,69$ 


\section{DA $1 / 98$}

Family Toxopneustidae

Nudechinus darnleyensis (Tenison-Woods,

1878)

N. scotiopremnus H.L. Clark, 1912

Tripneustes gratilla (Linnaeus, 1758)

Family Echinometridae

Echinostrephus molaris (Blainville, 1825)

\section{$3,4,8,12,13,15$,} $16,22,26,32$

\section{Family Clypeasteridae}

Clypeaster latissimus (Lamarck, 1816)

C. telurus H.L. Clark, 1914

\section{Family Laganidae}

Peronella lesueuri (Valenciennes, 1841)

P. macroproctes Koehler, 1922

P. orbicularis (Leske, 1778)

P. sp. cf. orbicularis Leske, 1778 )

P. tuberculata Mortensen, 1918

Peronella minuta (de Meijere, 1904)

Peronella sp. (incomplete)

Family Astriclypeidae

Echinodiscus auritus Leske, 1778

Fam. Echinolampadidae

Echinolampas ovata (Leske, 1778)

\section{Family Fibulariidae}

Fibularia sp. cf. acuta Yoshiwara, 1898

F. oblonga Gray, 1851

\section{Family Schizasteridae}

Schizaster(Schizaster) compactus (Koehler, 1914)
20

$11,14,20,23,25,31$
$73,76,91$

(n)

\section{$4,5,12,48,56$}

$6,9,23,32,41,49,58,71,72,76$

$1,4,18,29,32,35,37,39,40,41,47$,

$49,53,60,69,71,72,75,95$

$12,29,72,73,76,78$

$12,19,26,27,47$

$1,18,29,40,41,47,49,69,72,91,95$

7,72

$4,7,9,17,22,34,46,47,49,68,70,71,83,96$

$18,41,46,48,49,71$

46,78

$37,38,42$

38,47

51,54

$38,54,65$

\section{Family Brissidae}

Brissopsis sp. aff. persica Mortensen, 1940

Brissus latecarinatus (Leske, 1778)

Metalia sternalis (Lamarck, 1816)

Rhynobrissus hemiasteroides A. Agassiz, 1879 no loc.

$38,45,47,61,65,66,70$

$38,41,62,68$

83
68

cf. Rhynobrissus sp. (incomplete) 
Family Loveniidae

Breynia desorii Gray, 1851

$19,28,31$

Lovenia elongata (Gray, 1845)

11

\section{Family Spatangidae}

Nacospatangus (Pseudomaretia) interruptus (Studer, 1880)

\section{Class HOLOTHUROIDEA}

\section{Family Holothuriidae}

Actinopyga echinites (Jaeger, 1833)

Bohadschia marmorata (Jaeger, 1833)

Holothuria (Cystipus) inhabilis Selenka, 1867

H. (Halodeima) atra Jaeger, 1833

H. (Halodeima) edulis Lesson, 1830

H. (Lessonothuria) pardalis Selenka, 1867

$H$. (Lessonothuria) sp. juv.

H. (Mertensiothuria) leucospilota

(Brandt, 1835)

H. (Metriatyla) sp. aff. albiventer Semper, 1868

H. (Metriatyla) martensii Semper, 1868

H. (Metriatyla) scabra Jaeger, 1833

$H$. (Metriatyla) timana Lesson, 1830

H. (Platyperona) difficilis Semper, 1868

H. (Semperothuria) cinerascens (Brandt, 1835)

H. (Stauropora) fuscocinerea Jaeger, 1833

H. (Stauropora) modesta Ludwig, 1875

H. (Stauropora) pervicax Selenka, 1867

H. (Theelothuria) michaelseni Erwe, 1913

H. (Theelothuria) spinifera Théel, 1886

H. (Thymiosycia) arenicola Semper, 1868

H. (Thymiosycia) hilla Lesson, 1830

H. (Thymiosycia) impatiens (Forskål, 1775)

H. (Thymiosycia) sp

Holothuria sp.

Family Stichopodidae

Stichopus chloronotus Brandt, 1835

S. hermanni Semper 1868

S. horrens Selenka, 1867

S. sp. cf. horrens Selenka, 1867

S. sp.cf. monotuberculatus Quoy and

Gaimard, 1833

S. sp. cf. noctivagus Cherbonnier, 1980

S. variegatus group

$1,33,36,37,39,59,60,66,68,69$,

$70,71,72,75,76,91,94$

$4,7,24,31,46,47,51,52,53,71,78,83,91,96$

$33,46,49,57,58,62,76,89$

$42,57,68$

$37,40,42,44,45,47,48,53,66$

$42,44,45,49,51,56,57,64,66,70$

$36,42,44,47,49,50,51,52,58,64,67$ $36,45,49,51,53,59,60,62,63,64,67$

$51,59,62$

$37,42,45,47,48,51,54,56$

$58,59,64,66,70$

11

$2,11,16,22,23$

$6,7,8,12,14,16$

19

$19,22,25$

$14,19,22,28$

$10,19,23,25,28,35$

19

$3,6,10,11,16,32,35$

$12,23,24,31,33,35$

29

$6,49,71$

$3,16,20,34,40$

49,70

70,96

$5,21,27,36$

57,75

$42,45,47,49,62$

$38,42,44,45,48,54$

38

\section{$42,44,45,51,56,58$}

54,57

$42,44,45,51,56,61,64$ 
Stichopus sp. juv.

\section{Family Cucumariidae}

Actinocucumis typica Ludwig, 1875

Cercodemas anceps Selenka, 1867

Colochirus crassus Ekman, 1918

C. quadrangularis Troschel, 1846

Leptopentacta grisea H.L. Clark, 1938

Leptopentacta sp.

Loisettea amphictena Rowe and Pawson, 1985

L. gazellae (Lampert, 1889)

Mensamaria intercedens (Lampert, 1885)

Plesiocolochirus australis (Ludwig, 1875)

$P$. challengeri (Théel, 1886)

Pseudocolochirus violaceus (Théel, 1886)

\section{Family Phyllophoriidae}

Havelockia versicolor (Semper, 1868)

Hemithyone semperi (Bell, 1884)

Neothyonidium magnum (Ludwig, 1882)

Phyllophorus (Phyllophorella) spiculata

$$
\text { Chang, } 1935
$$

P. (Urodemella) brocki Ludwig, 1888

P. (Urodemella) proteus Bell, 1884

Phyrella sp. cf. trapeza (H.L. Clark, 1932)

Stolus buccalis (Stimpson, 1855)

S. canescens (Semper, 1868)

S. conjungens (Semper, 1868)

Thyone dura Koehler and Vaney, 1908

T. grisea H.L. Clark, 1938

Thyone papuensis Théel, 1886

Thyone sp. cf. papuensis Théel, 1886

Thyone sp. aff. theeli Rowe, 1995

Thyone sp.

Gen. and sp. indet. (1)

Gen. and sp. indet. (2)

Family Sclerodactylidae

Afrocucumis africana (Semper, 1868)

Cladolabes schmeltzii (Ludwig, 1875)

Family Caudinidae

$$
57
$$

$1,16,24,27,32$

20,29

69

8

57

10

55
26

10

21

8
$7,31,59,72$

$1,4,12,18,21,25,33,39,50,59,67,70,75$

$8,10,21,50,55,57,75$

$8,9,10,18,37,39,59,65,68,69,70,75$

$45,47,70$

$4,6,21,24,33,75,96$

$44,45,47,56$

$4,10,44,59,70$

$6,8,9,10,50,72$

$6,12,21,22,44,79$

$39,49,50$

$52,55,57,65$

$4,8,10,50,75,94$

10,59

59,94

$33,37,50,65,70$

$39,42,65,68,69,75,98$

$4,6,9,37,57,79$

39,55

4,12 
Acaudina leucoprocta (H.L. Clark, 1938)

A. sp. cf. molpadioides (Semper, 1868)

$1,16,19,24,34,49,61,96$

Family Molpadiidae

Molpadia scabrum (Sluiter, 1901)

$23,28,34,35,55,90$

Family Synaptidae

Synaptula macra (H.L. Clark, 1938)

S. recta (Semper, 1868)

Synaptula sp. (incomplete)
$5,12,22$
$6,8,9,10,37,39,59,62,65,68,72,75$
$41,55,62$ 
Appendix 2 Echinoderm species not found during the WA Museum-Woodside Survey but recorded from specimens in the WA Museum from earlier work in the Dampier Archipelago, to 40 m depth.

\section{Class CRINOIDEA}

Family Comasteridae

Capillaster multiradiatus (Linnaeus, 1758) $19 \mathrm{~m}, \mathrm{E}$ of Delambre I.

\section{Class ASTEROIDEA}

Family Oreasteridae

Anthenea godeffroyi Doderlein, 1915

$37 \mathrm{~m}$, between Gidley and Rosemary Is

$18 \mathrm{~m}$, between Malus and Gidley Is

Anthenea pentagonula (Lamarck, 1816)

$9 \mathrm{~m}$, Mermaid Strait

Anthenea sibogae Doderlein, 1915

$42 \mathrm{~m}, 2 \mathrm{n}$. miles W of Legendre I.

Family Ophidiasteridae

Linckia guildingi Gray, 1840

Family Echinasteridae

Echinaster luzonicus (Gray, 1840)

$18 \mathrm{~m}, 3-4 \mathrm{n}$. miles E of Delambre I.

\section{Class OPHIUROIDEA}

Family Gorgonocephalidae

Astrochalcis tuberculosus Koehler, 1905 $40 \mathrm{~m}, 8 \mathrm{n}$. miles $\mathrm{N}$ of Delambre I.

Family Amphiuridae

Amphioplus (Lymanella) hastatus (Ljungman, 1867) 7-9 m, W of Conzinc I.; 1-2 m, Norbill Bay, Rosemary I.

Amphiura (Amphiura) leucaspis H.L. Clark, 1938 1-2 m, Norbill Bay, Rosemary I.

A. (Amphiura) micra H.L. Clark, 1938 $12 \mathrm{~m}, \mathrm{~W}$ of Gidley I.; 13-16 m SW of Hamersley Shoal

A. (Amphiura) cf. microsoma H.L. Clark, 1915 Intertidal, Rosemary I.

A. (Amphiura) stictacantha H.L. Clark, 1938 Intertidal, Rosemary I.

A. (Fellaria) octacantha (H.L. Clark, 1915) 0-10 m, Rosemary I.

A. (Ophiopeltis) tenuis (H.L. Clark, 1938) 0-1 m, Hearson Cove, Burrup Peninsula

\author{
Family Ophiotrichidae \\ Gymnolophus obscura (Ljungman, 1867) \\ 1-10 m, Kendrew I. \\ Family Ophiocomidae \\ Ophiocoma erinaceus Müller and Troschel, 1842 \\ 0-1 m, Kendrew I. \\ Ophiocoma pica Müller and Troschel, 1842 \\ 0-1 m, Kendrew I. \\ Family Ophiodermatidae \\ Cryptopelta callista H.L. Clark, 1938 \\ Inter-tidal, Kendrew I. \\ Ophiochaeta hirsuta Lütken, 1869 \\ 0-1 m, Kendrew I.

\section{Class ECHINOIDEA} \\ Family Echinothuriidae \\ Asthenosoma ijimai Yoshiwara, 1897 \\ 32-36 m, NW of Delambre I. \\ Family Diadematidae \\ Astropyga radiata (Leske, 1778) \\ $43 \mathrm{~m}, 11 \mathrm{n}$. miles NNE of Legendre I. \\ Diadema savignyi (Michelin, 1845) \\ $6 \mathrm{~m}$, Kendrew I. \\ Echinothrix calamaris (Pallas, 1774) \\ $6 \mathrm{~m}$, Kendrew I. \\ Family Echinometridae \\ Echinometra mathaei (Blainville, 1825) \\ Intertidal, Delambre I.; Kendrew I.

\section{Class HOLOTHUROIDEA} \\ Family Holothuriidae \\ Labidodemas semperianum Selenka, 1867 \\ 0-1 m, Kendrew I. \\ Family Synaptidae \\ Rynkatorpa sp. cf. bisperforata (H.L. Clark, 1938) \\ 0-1 m, Rosemary I.
}

